\title{
An extended flame index partitioning for partially premixed combustion
}

\author{
Enric Illana ${ }^{\mathrm{a}}$ and Daniel Mira ${ }^{\mathrm{b}}$ and Arnaud Mura ${ }^{\mathrm{c}}$ \\ ${ }^{a}$ Department of Energy Plant Technology (LEAT), Ruhr-University Bochum, \\ Universitätsstraße 150, 44780 Bochum, Germany; \\ ${ }^{b}$ Barcelona Supercomputing Center (BSC), C/ Jordi Girona 29, 08034 Barcelona, Spain; \\ ${ }^{c}$ Institut PPRIME - UPR 3346 - CNRS - ISAE-ENSMA - Université de Poitiers \\ BP 40109, 86961 Futuroscope, France
}

(Compiled December 8, 2020)

\begin{abstract}
Compared to partially premixed combustion (or combustion of non-homogeneous reactants in general), fully premixed and diffusion flames represent only two asymptotic limits of combustion modes. However, the deep knowledge accumulated over the years on these two elementary and archetypal flame prototypes is such that they remain the cornerstone and reference building blocks of most combustion modelling proposals. Therefore, from a general point of view, being able to distinguish between premixed and non-premixed modes of combustion thanks to a flame index appears as a quite appealing but challenging task that still concentrates many research efforts. Indeed, the availability of such an index is not only appealing to proceed with the analysis of either experimental or computational data issued from DNS (or highly resolved LES) databases. It is also an essential ingredient to elaborate advanced flamelet-based multiregime combustion models on the basis of single regime tabulated flamelet databases. In the present study, a new definition of the premixedness index $\zeta_{\mathrm{PF}}$ is proposed for partially premixed combustion. It is based on a weighted form of the cross-scalar dissipation rate of the mixture fraction $Y_{\xi}$ and progress variable $Y_{c}$, i.e., quantities that have been previously identified as relevant parameters to describe partially premixed combustion regimes. The relevance of the corresponding index is assessed through a detailed computational procedure that includes three successive validation subsets: counterflow flames (including premixed, rich partially-premixed, and diffusion flames), (ii) stabilized triple flames for three distinct values of the inlet mixture fraction gradient, and finally (iii) unsteady flame kernel developments in nonhomogeneous mixtures of fresh reactants, which are characterized by various initial levels of the segregation rate between the fuel and oxidizer. The proposed premixedness index $\zeta_{\mathrm{PF}}$ and its counterpart $\zeta_{\mathrm{DF}}=1-\zeta_{\mathrm{PF}}$ are used as the weighting coefficients between tabulated premixed flamelets (TPF) and tabulated diffusion flamelets (TDF) data, which have been parameterized as functions of $Y_{\xi}$ and $Y_{c}$. It is noteworthy that, in contrast to some previous proposals of the literature, the present flame index does not require the consideration of any other quantities in addition to those already used to parameterize the flamelets databases, i.e., $Y_{\xi}$ and $Y_{c}$. The validation procedure makes use of steady and unsteady processes with a priori and a posteriori analyses. In both cases, the comparisons between the results obtained with the proposed flame partitioning and detailed chemistry (DC) computations lead to a satisfactory level of agreement and, from a general viewpoint, the level of agreement is better than the one obtained with either premixed or diffusion flamelet-based models.
\end{abstract}

Keywords: premixed combustion; non-premixed combustion; partially premixed combustion; flame index; premixedness index; multi-regime flamelet model 


\section{Introduction and general context}

Consideration of detailed chemistry effects remains one of the most important challenges in numerical combustion because it is intrinsically computational demanding. This specific issue has motivated the development of simplified chemical schemes recovering some essential features of chemistry and laminar flames, e.g., ignition delays, laminar premixed flame propagation velocity and thickness, among others, see for instance references [1-4]. Detailed chemistry effects can however be considered through other strategies, which make use of tabulated chemistry models. They consist in proceeding with a direct a priori mapping of the detailed chemistry response of the flame onto a reduced set of characteristic variables to account for detailed chemistry effects at a reasonable computational cost. Any quantity $\varphi$ of interest (e.g., chemical source terms, molecular transport properties, and coefficients of the NASA polynomials as it is proposed herein) is stored in the corresponding look-up tables. There exist various methodologies to generate such chemical look-up tables. For instance, flamelet generated manifold (FGM) or flame-prolongated ILDM (FPI) are very popular [5]6] and have been previously used to describe partially premixed combustion [7|8]. They rely on tabulated premixed flamelets (TPF) and assume that the chemistry response of partially premixed flames (PPF) can be described on the basis of a reduced phase subspace $\left(Y_{\xi}, Y_{c}\right)$ associated to fully premixed flames structures computed for various values of the equivalence ratio $\Phi$. In general, a mixture fraction $Y_{\xi}$ can be used to describe the fuel/air mixture ratio and a progress variable $Y_{c}$ is defined to represent the chemical evolution. Different proposals can be found in the literature and the retained definition may have a certain impact on the model performance. Standard definitions rely on a combination of some chemical species mass fractions such as a sum of carbon monoxide and carbon dioxide mass fractions, i.e., $Y_{c}=0.5 \cdot\left(Y_{\mathrm{CO}}+Y_{\mathrm{CO} 2}\right)$ [8], or can be deduced from an optimization procedure [9[10]. Tabulated diffusion flamelets (TDF) have been also early considered as a possible building block [11] and computations of nonpremixed counterflow flames in the physical space can be used to map the detailed chemistry response as a function of the mixture fraction $Y_{\xi}$ and progress variable $Y_{c}$ [12]13]. Another strategy may consist in considering directly a collection of laminar one-dimensional partially premixed flamelets. For instance, Franzelli et al. [14] considered the injection of pure air against a fuel/air mixture for different values of the equivalence ratio $\Phi$. To account for the possible premixing of fuel on the odixizer side, Wen et al. [15] recently extended this proposal to the consideration of counterflow PPF featuring a lean stream of methane and air opposed to a rich stream of methane and air. The consideration of such tabulated partially premixed flamelets (TPPF) as the elementary building block of the tabulation offers an interesting possibility, which avoids the blending (or partitioning) procedure. This is however at the price of larger look-up tables. For instance, in Wen et al. [15], the thermo-chemical quantities $\varphi$ are parameterized as functions of four scalar quantities $\left(Y_{\xi}, Y_{c}, Y_{O}, Y_{F}\right)$, with two additional trajectory variables $Y_{O}$ and $Y_{F}$ used to track the partial premixing of the fuel and oxidizer streams, respectively. The use of such tabulations in turbulent conditions requires further work since the joint PDF of the corresponding quantities must be approximated, and this may require to consider not only a closed set of modelled transport equations for their filtered values, but also many additional cross-correlations between subgrid scalar fluctuations. However, the corresponding modelling issues are outside the scope of the present work, which conditions.

To conclude, it should be emphasized that the elementary building blocks mentioned above, i.e., TPF, TDF, and TPPF, share some common limitations and there exist several directions for further improvements. For instance, since the tabulated data are based on onedimensional computations, the spatial variations of mixture fraction and progress variable are 
necessarily aligned in either one-dimensional TDF or one-dimensional TPPF building blocks, whereas they are not systematically oriented along the same direction in practical situations. However, the use of two-dimensional laminar partially premixed flames as the elementary building block would raise so complicated issues that this strategy is not considered as relevant for future developments. In this respect, it is noteworthy that the definition of a blending function able to account for the possible influence of a misalignment between the gradients of $Y_{\xi}$ and $Y_{c}$ would be extremely beneficial. This special point will be discussed in more details in the next section of this work. In addition to this, it should be emphasized that, over the years, a large amount of knowledge has been accumulated to develop and construct TPF and TDF databases that reproduce the burning rates and chemical species formation with a remarkable level of accuracy [8|9|14|16|17]. A large number of these databases have been gathered for various operating conditions and there is therefore some undeniable interest in finding an appropriated partitioning function to take advantage of their immediate availability.

The present manuscript is organized as follows: in the next section, a generalized premixedness index is introduced to distinguish between premixed and non-premixed modes of combustion. Its relevance is first evaluated from the inspection of counterflow partially premixed flames in the third section. Since the present work is primarily concerned with general situations, where the existence of both fuel-rich and fuel-lean pockets may lead to a combination of premixed and diffusion flame burning, the archetypical laminar partially premixed flame, i.e., the two-dimensional triple flame, is then considered as a relevant test case in section 4. The same modelling strategy is subsequently used to perform the computations of flame kernel developments in non-homogeneous mixtures of fresh reactants. Finally, the paper ends with a conclusion section where some perspectives for future works are presented.

\section{Flame indexes and flame partitioning}

\subsection{Existing definitions of the flame index}

Over the last twenty-five years, there is a large amount of strategies that have been proposed in the literature so as to determine the local burning modes, i.e., premixed or non-premixed. Some of these methods do account explicitly for detailed chemistry effects, as with the chemical explosive mode analysis (CEMA), see for instance reference [18]19], whereas some others do not, as with the flame index (FI) concept, which was early introduced by Yamashita et al. [20] in their numerical study of jet diffusion flame stability. Instead the latter (i.e., the FI) is based on the consideration of molecular diffusion fluxes in one-dimensional premixed and diffusion flame structures. DNS studies nonetheless showed that it may be relevant for multidimensional situations [21|22].

This index is often referred to as the Takeno index, it is denoted by $G_{F O}$ and its expression reads:

$$
G_{F O}=\nabla Y_{F} \cdot \nabla Y_{O}
$$

where $Y_{F}$ and $Y_{O}$ correspond to the fuel and oxidizer mass fractions, respectively. It is positive in a one-dimensional fully premixed flame, whereas it is negative for a diffusion flame. It can be readily rewritten in terms of the unit vectors normal to the fuel and oxidizer iso-lines:

$$
G_{F O}=\left|\boldsymbol{\nabla} Y_{F}\right|\left|\boldsymbol{\nabla} Y_{O}\right| \quad\left(\boldsymbol{n}_{F} \cdot \boldsymbol{n}_{O}\right)=\left|\boldsymbol{\nabla} Y_{F}\right|\left|\boldsymbol{\nabla} Y_{O}\right| \cos \left(\boldsymbol{n}_{F}, \boldsymbol{n}_{O}\right)
$$

with $\boldsymbol{n}_{F}=\boldsymbol{\nabla} Y_{F} /\left|\boldsymbol{\nabla} Y_{F}\right|$ and $\boldsymbol{n}_{O}=\boldsymbol{\nabla} Y_{O} /\left|\boldsymbol{\nabla} Y_{O}\right|$. 
Moreover, under the unity Lewis number assumption, it can also be expressed as a function of the cross scalar dissipation rate (SDR) of fuel and oxidizer $\mathscr{L}_{F O}$

$$
G_{F O}=\mathscr{D}^{-1} \mathscr{L}_{F O}
$$

where $\mathscr{D}$ denotes the molecular diffusivity.

The expression given by Eq. (2) clearly shows that, as it is defined, such an index is nothing but a measure of the orientation (i.e., the direct cosine) between the two gradients of fuel and oxidizer mass fractions. In this respect, depending on this orientation, the cross SDR, i.e., $\mathscr{L}_{F O}=\mathscr{D} \boldsymbol{\nabla} Y_{F} \cdot \nabla Y_{O}$, plays the role of either a sink $\left(\mathscr{L}_{F O}>0\right)$ or a source $\left(\mathscr{L}_{F O}<0\right)$ term in the transport equation of $Y_{F} Y_{O}$ (remembering that it appears with a minus sign in the corresponding budget).

From a more general point of view, a normalized premixedness index can also be directly deduced from the following expression [23]:

$$
\zeta_{\mathrm{PF}} \equiv \frac{1}{2}\left(1+\frac{\mathbb{V}_{F}}{\left|\mathbb{V}_{F}\right|} \cdot \frac{\mathbb{V}_{O}}{\left|\mathbb{V}_{O}\right|}\right)
$$

where $\mathbb{V}_{\alpha}$ denotes the molecular diffusion velocity of the fuel $(\alpha=F)$ or oxidizer $(\alpha=O)$. Its values are zero and unity in the limits of diffusion flame and fully premixed combustion, respectively. The above expression can also be written as follows: $\zeta_{\mathrm{PF}}=\left(1+\boldsymbol{n}_{F} \cdot \boldsymbol{n}_{O}\right) / 2$, where the unit direction $\boldsymbol{n}_{\alpha}=\mathbb{V}_{\alpha} /\left|\mathbb{V}_{\alpha}\right|$ corresponds now to the directions of the molecular diffusion fluxes of chemical species $\alpha$. Thus, the above expression, i.e., the one given by Eq. (4), may be retained to circumvent the assumption of the Fick's law of diffusion.

If Fickian diffusion is however assumed, the unit vector $\boldsymbol{n}_{\alpha}$, which gives the direction of the molecular diffusion flux of species $\alpha$, may be directly expressed from the species mass fraction gradients: $\boldsymbol{n}_{\alpha}=\boldsymbol{\nabla} Y_{\alpha} /\left|\nabla Y_{\alpha}\right|$, which allows to relate the premixedness index $\zeta_{\mathrm{PF}}$ to the flame index:

$$
\zeta_{\mathrm{PF}}=\frac{1}{2}(1+\Psi)
$$

where the definition of the normalized flame index $\Psi$ has been introduced

$$
\Psi=\frac{G_{F O}}{\left|\nabla Y_{F}\right| \cdot\left|\nabla Y_{O}\right|}
$$

At this level, since some errors have been previously propagated in the literature, it seems worthwhile to emphasize that the objective of all the above index definitions is to compare molecular fluxes orientations. Therefore, the denominator in the above equation, i.e., Eq. (6), should not be $\left|\nabla Y_{F} \cdot \nabla Y_{O}\right|$ but must indeed be $\left|\nabla Y_{F}\right| \cdot\left|\nabla Y_{O}\right|$, which is nothing but the consequence of the unit vectors definition: $\boldsymbol{\nabla} Y_{F}$ is normalized by $\left|\nabla Y_{F}\right|$ and $\boldsymbol{\nabla} Y_{O}$ by $\left|\nabla Y_{O}\right|$. In this manuscript, the expression given by Eqs. (5) and (6) will be referred to as TPI, i.e., Takeno premixedness index.

However, there are other issues associated to the use of the above expressions, i.e., Eq. (1), Eq. (4) or Eq. (5). For instance, comparing the directions of the fuel and oxidizer molecular fluxes seems suitable for single step chemistry, though it may become less representative within the framework of detailed chemistry where other species than fuel may be relevant. For instance, intermediate species such as $\mathrm{CO}$ and $\mathrm{H}_{2}$ play an essential role in fuel-rich regions. Long chained primary fuel molecules may completely dissociate well before they reach the flame reaction zone and, as a consequence, the primary fuel mass fraction gradient may 
become irrelevant. There were therefore some attempts to generalize the existing definitions by considering the mass fractions of unburnt carbon and oxygen atoms instead of fuel and oxidizer [24]

$$
Y_{F} \equiv \sum_{\alpha}^{\alpha \neq \mathrm{CO}_{2}, \mathrm{H}_{2} \mathrm{O}} \frac{\mathcal{M}_{\mathrm{C}}}{\mathcal{M}} Y_{\alpha}, Y_{O} \equiv \sum_{\alpha}^{\alpha \neq \mathrm{CO}_{2}, \mathrm{H}_{2} \mathrm{O}} \frac{\mathcal{M}_{\mathrm{O}}}{\mathcal{M}} Y_{\alpha}
$$

where $\mathcal{M}$ denotes the molecular weight of the multi-component mixture and $\mathcal{M}_{\alpha}$ the one of chemical species $\alpha$.

The definition of the flame index provided above, see Eq. (1), as well as some generalized forms which were derived from its original expression, see Eq. (4) or Eq. (5), remain still very popular today [23-26]. However, previous analyses from Fiorina et al. [8] and Knudsen and Pitsch [16] put some of their limitations into evidence. For instance, as documented in reference [8], in the case of rich partially premixed flames, some discrepancies may occur in the transition zone between premixed and diffusion modes, where the burning rates may remain diffusion-controlled even though fuel and oxidizer gradients are pointing towards the same direction. Some discrepancies have been also identified through the detailed analysis of triple flame simulations [16]. It is therefore proposed to introduce a new definition of the flame index.

\subsection{A proposal for a new flame index}

Before embarking on the introduction of such a proposal, it seems worthwhile to remind that flamelet databases are generally parameterized as functions of $Y_{\xi}$ and $Y_{c}$. Therefore, to keep pace with a reduced number of variables, an optimal choice would consist in expressing the flame index as a function of these two variables only. In this respect, it is also noteworthy that the orientation between the mixture fraction and progress variable gradients, as well as their associated amplitudes, have been early identified as relevant parameters to characterize combustion of non-homogeneous reactants [27/28]. From this point of view, the mixture fraction gradient is aligned with the progress variable gradient across a local one-dimensional diffusion flame. Therefore, to assess the possible relevance of tabulated diffusion flamelets (TDF), the flame index should evaluate the orientation between the direction of mixture fraction gradient $\boldsymbol{n}_{Y_{\xi}}=\boldsymbol{\nabla} Y_{\xi} /\left|\boldsymbol{\nabla} Y_{\xi}\right|$ and the one of the progress variable gradient $\boldsymbol{n}_{Y_{c}}=\boldsymbol{\nabla} Y_{c} /\left|\boldsymbol{\nabla} Y_{c}\right|$. Moreover, the magnitude of the corresponding mixture fraction gradient $\left|\nabla Y_{\xi}\right|$ can be normalized with its tabulated counterpart $\left|\nabla Y_{\xi}\right|^{\mathrm{TDF}}\left(Y_{\xi}, Y_{c}\right)$.

The following proposal fulfills these two objectives:

$$
\begin{aligned}
\zeta_{\mathrm{PF}} & =\left(1-\frac{\left|\nabla Y_{\xi}\right|}{\left|\nabla Y_{\xi}\right|^{\mathrm{TDF}}\left(Y_{\xi}, Y_{c}\right)} \boldsymbol{n}_{Y_{\xi}} \cdot \boldsymbol{n}_{Y_{c}}\right) \\
& =\left(1-\frac{\mathscr{L}_{Y_{\xi} Y_{c}}}{\mathscr{D}\left|\boldsymbol{\nabla} Y_{c}\right|\left|\boldsymbol{\nabla} Y_{\xi}\right|^{\mathrm{TDF}}\left(Y_{\xi}, Y_{c}\right)}\right)
\end{aligned}
$$

where $\mathscr{L}_{Y_{\xi} Y_{c}}=\mathscr{D} \nabla Y_{\xi} \cdot \nabla Y_{c}$ denotes the the cross-scalar dissipation rate.

It is noteworthy that, in the above expression, the dot product between $\boldsymbol{n}_{Y_{\xi}}$ and $\boldsymbol{n}_{Y_{c}}$ is nothing but a measure of the misalignment between the mixture fraction and progress variable gradients: it is the cosine of the angle between these two directions. Thus, in contrast to the early proposal of Takeno and his coworkers, (i) the above expression does involve the mixture fraction gradient and (ii) it takes into account the possible misalignment between the 
mixture fraction and progress variable gradients. It seems worth emphasizing that, in moderately curved diffusion flames, the mixture fraction and progress variable gradients are aligned and the dot product $\boldsymbol{n}_{Y_{\xi}} \cdot \boldsymbol{n}_{Y_{c}}$ is unity, whereas in premixed or weakly stratified flames, this dot product tends to be vanishingly small. In this respect, with the objective of discriminating combustion modes, the dot product $\boldsymbol{n}_{Y_{\xi}} \cdot \boldsymbol{n}_{Y_{c}}$ has been previously applied, as a post-processing procedure, to the filtered fields of the mixture fraction and progress variable issued from LES computations [29]. However, the direct use of the dot product $\boldsymbol{n}_{Y_{\xi}} \cdot \boldsymbol{n}_{Y_{c}}$ would lead the values of he corresponding premixedness index, i.e., $\zeta_{\mathrm{PF}}=\left(1-\boldsymbol{n}_{Y_{\xi}} \cdot \boldsymbol{n}_{Y_{c}}\right)$, to be zero in one-dimensional partially premixed flames such as those considered in section 3.2. Thus, such a definition would be unable to account for partial premixing effects. This is in contrast with the expression given by Eq. (8), where the dot product is weighted by the normalized magnitude of the mixture fraction gradient, i.e., $\left|\nabla Y_{\xi}\right| /\left|\nabla Y_{\xi}\right|^{\mathrm{TDF}}\left(Y_{\xi}, Y_{c}\right)$.

From a practical point of view, the above definition of the premixedness index can be rewritten in a simpler way:

$$
\zeta_{\mathrm{PF}}=\left(1-\frac{\boldsymbol{\nabla} Y_{\xi} \cdot \boldsymbol{n}_{Y_{c}}}{\left|\boldsymbol{\nabla} Y_{\xi}\right|^{\mathrm{TDF}}\left(Y_{\xi}, Y_{c}\right)}\right)
$$

On the hand, it is noteworthy that $\left|\nabla Y_{\xi}\right|^{\mathrm{TDF}}$ is obtained from the reference one-dimensional strained diffusion flame solution, a situation where the mixture fraction gradient is aligned with the progress variable gradient. On the other hand, the dot product $\boldsymbol{\nabla} Y_{\xi} \cdot \boldsymbol{n}_{Y_{c}}$ denotes the component of the mixture fraction gradient vector in the direction normal to the progress variable iso-lines. Thus, only the component of the mixture fraction gradient that is aligned with the progress variable gradient is considered. In fact, the proposed index is defined so that the mixture fraction gradient is normalized by the tabulated value to evaluate the strength of the corresponding gradient, and then projected onto the progress variable gradient direction, which evaluates that misalignment.

As it is formulated, the above expression of the premixedness index is however lacking chemical inputs, whereas the influence of the lean and rich flammability limits of premixed flamelets should be addressed. One of the simplest way to take them into account consists in introducing a correction factor $k$ :

$$
\zeta_{\mathrm{PF}}=k\left(1-\frac{\boldsymbol{\nabla} Y_{\xi}}{\left|\boldsymbol{\nabla} Y_{\xi}\right|^{\mathrm{TDF}}\left(Y_{\xi}, Y_{c}\right)} \cdot \frac{\boldsymbol{\nabla} Y_{c}}{\left|\boldsymbol{\nabla} Y_{c}\right|}\right)
$$

the value of which is set to unity $(k=1)$ within the flammability region while, outside of this region, it is linearly interpolated to zero at $Y_{\xi}=0$ and $Y_{\xi}=1$.

Before proceeding with the computational study of this flame index, it should be emphasized that its derivation has also been guided by simplicity, which is not only an important quality of any model but also a necessary requirement in prevision of its future applications to turbulent reactive flows simulations. In view of this simplicity, it seems worthwhile to conclude this section by describing the possible limitations that may be expected from the use of such an index. As a preliminary remark, it could be argued that premixed flames may propagate across a transverse gradient of equivalence ratio but this requires the corresponding gradient to remain rather moderate to avoid possible extinction. It is also noteworthy that premixed flames can also propagate along the same direction as an equivalence ratio gradient [30] and, as it is formulated, it seems that the above formulation could be thought unable to identify such a situation as a premixed combustion mode. However, it should be noted that this is fully consistent with the elementary building blocks that are considered: the tabulated one-dimensional fully premixed flames indeed ignore any possible variations of the equiv- 
alence ratio. Moreover, we will see that the computations of flame kernel growths in non homogeneous mixtures of reactants lead to quite satisfactory results. This may be explained as follows: premixed flame propagation in stratified or non homogeneous media has been indeed observed but only for rather moderate values of the mixture fraction gradients, which are significantly smaller than the reciprocal of the laminar premixed flame thickness $1 / \delta_{L}^{0}$. Mixture fraction variations thus remain quite negligible over length scales that are typical of the laminar flame thickness. Such conditions correspond to quite small values of the ratio $\left|\nabla Y_{\xi}\right| /\left|\nabla Y_{\xi}\right|^{\mathrm{TDF}}\left(\xi, Y_{c}\right)$, in such a manner that the premixedness index value remains close to unity, see Eq. (11). The partially premixed flame can be considered as a collection of fully premixed local flames.

In comparison with detailed chemistry computations that do involve the transport of hundreds of chemical species, computations based on tabulated data thus proceed only with the resolution of the following additional scalar transport equations:

$$
\begin{aligned}
& \mathcal{L}\left(\rho Y_{\xi}\right)=\frac{\partial \rho Y_{\xi}}{\partial t}+\boldsymbol{\nabla} \cdot(\rho \boldsymbol{u} \xi)-\boldsymbol{\nabla} \cdot\left(\rho \mathscr{D} \nabla Y_{\xi}\right)=0 \\
& \mathcal{L}\left(\rho Y_{c}\right)=\frac{\partial \rho Y_{c}}{\partial t}+\boldsymbol{\nabla} \cdot\left(\rho \boldsymbol{u} Y_{c}\right)-\boldsymbol{\nabla} \cdot\left(\rho \mathscr{D} \nabla Y_{c}\right)=\dot{\omega}_{Y_{c}}
\end{aligned}
$$

where $\mathcal{L}$ denotes the conservative transport operator and $\dot{\omega}_{Y_{c}}$ the production rate of the progress variable.

From the knowledge of the values of the mixture fraction $Y_{\xi}$, progress variable $Y_{c}$, and premixedness index $\zeta_{\mathrm{PF}}$, this contribution may be determined from the premixed and diffusion flamelets libraries,

$$
\dot{\omega}_{Y_{c}}=\zeta_{\mathrm{PF}} \cdot \dot{\omega}_{Y_{c}}^{\mathrm{TPF}}\left(Y_{\xi}, Y_{c}\right)+\zeta_{\mathrm{DF}} \cdot \dot{\omega}_{Y_{c}}^{\mathrm{TDF}}\left(Y_{\xi}, Y_{c}\right)
$$

with $\zeta_{\mathrm{DF}}=1-\zeta_{\mathrm{PF}}$. In the above expression, $\dot{\omega}_{\alpha}^{\mathrm{TPF}}\left(Y_{\xi}, Y_{c}\right)$ and $\dot{\omega}_{Y_{c}}^{\mathrm{TDF}}\left(Y_{\xi}, Y_{c}\right)$ denote the progress variable production rates that come from the premixed and diffusion flamelets libraries, respectively. As emphasized in the previous section, one of the main deficiencies of the widely used tabulation methods is that they generally assume a single combustion mode. Even if the use of a blending procedure remains difficult to justify from a physical viewpoint, a proposal such as the one presented above has the potential to circumvent this limitation by combining tabulated diffusion flamelets (TDF) with tabulated premixed flamelets (TPF). Aside from the intrinsic shortcomings of any blending procedures, it seems worth emphasizing that the proposal of an improved flame index for the the analysis of partially premixed conditions remains a very relevant and challenging issue for combustion theory and modelling. In fact, from a fundamental viewpoint, proceeding with the analysis of partially premixed combustion still remains a quite difficult task and, in this respect, it is noteworthy that, whatever experimental or computational data are considered, one of the starting point of such analyses generally consists in discriminating premixed from non-premixed regions [23|31--34]. Despite its limitations, such combustion modes analyses remain often based on the use of the Takeno index [23|31-33]. The modified premixedness index that is proposed herein may provide an improved framework to conduct such analyses while keeping one of the most attractive features of the Takeno index compared to other strategies, namely its simplicity. However, the relevance of the premixedness index $\zeta_{\mathrm{PF}}$ is a crucial issue. This is the subject of the next sections of this manuscript, which are devoted to its assessment in various conditions 


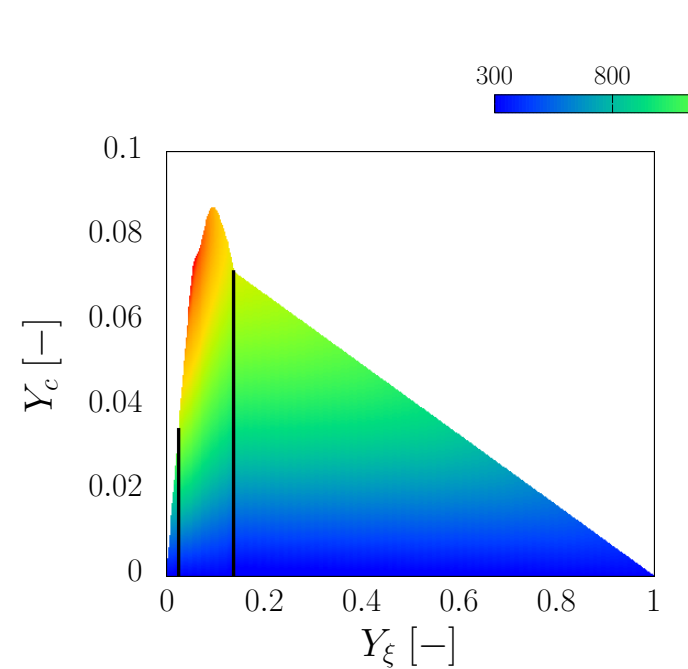

$T[K]$
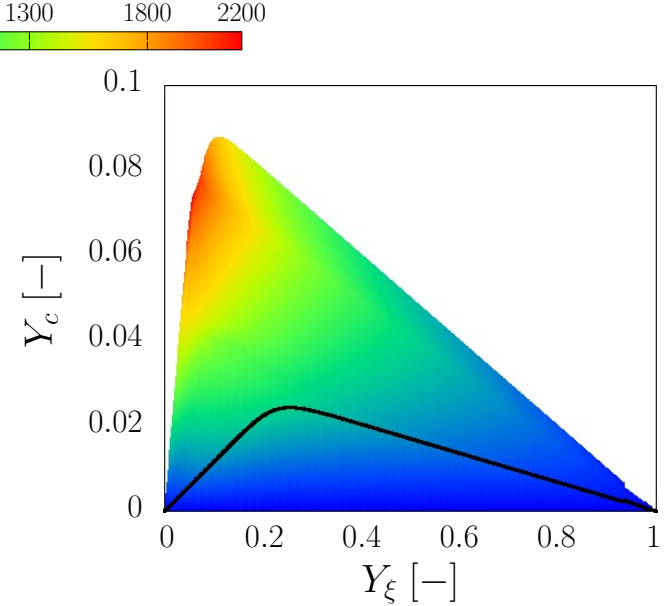

$\dot{\omega}_{Y_{c}}\left[k g /\left(m^{3} s\right)\right]$

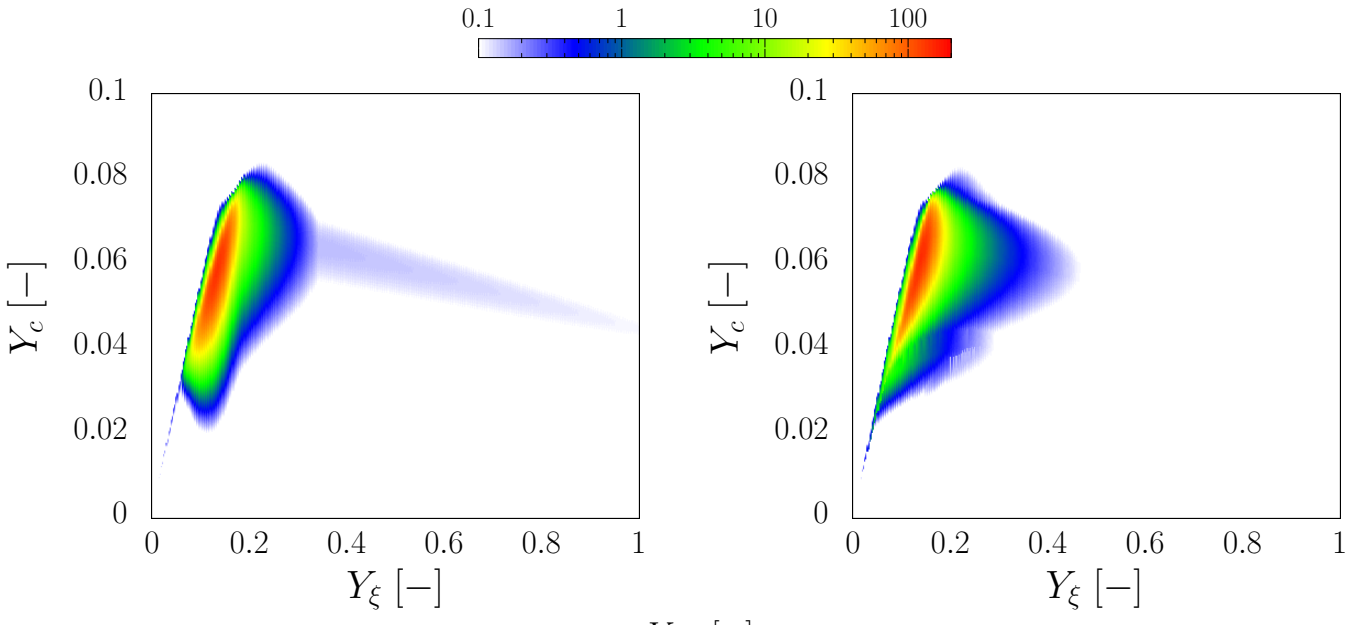

$Y_{\mathrm{CO}}[-]$

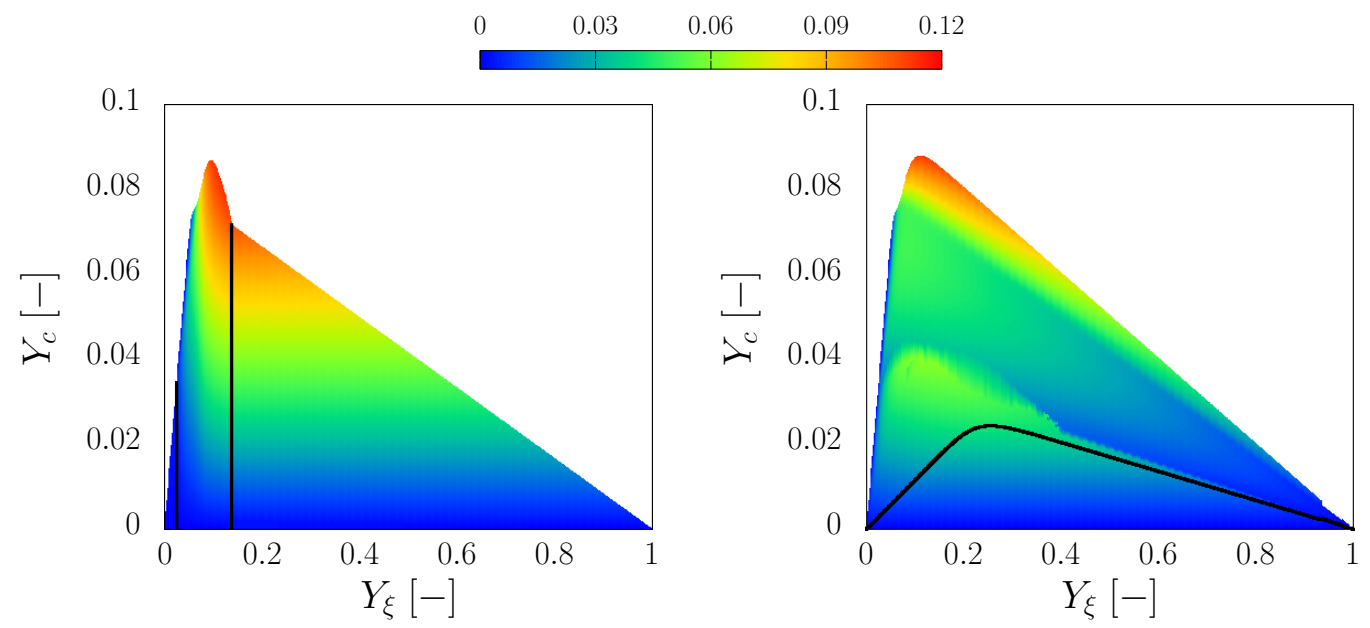

Figure 1.: Temperature (top), progress variable source term (middle), and CO mass fraction contours (bottom) obtained using premixed (left) and diffusion (right) flamelet prototypes. 


\subsection{Flamelet databases}

In the forthcoming sections of the manuscript we analyze the relevance of the model proposed above. This requires the use of flamelet manifolds, which are generated by using the Cantera software [35]. Methane-air combustion is described with the San Diego mechanism [36]. Non-unity Lewis number effects are not addressed just for the sake of simplicity and to make easier the comparisons between tabulated flame computations and detailed chemistry results.

The flamelet manifolds are standardly parameterized by means of two reference scalar variables [13]. On the one hand, the fresh mixture composition variations are described using a mixture fraction variable $Y_{\xi}$ [11] and the definition of Bilger [37] has been retained to proceed with the construction of the tabulated databases. The proportion of mass issued from the fuel inlet stream is given by $Y_{\xi}$, while that issued from the oxidizer inlet stream is $\left(1-Y_{\xi}\right)$. In this respect, since only two inlet stream situations are considered, the mixture fraction corresponds to a fuel inlet tracer that gives the local amount of mass issued from the inlet streams [38]. In this respect, it is worth recalling that, in this case, mixture fraction definitions are all equivalent, i.e., the mixture fraction can be defined as any combination of chemical species mass fractions provided that it ensures a passive scalar behavior - based on the conservation of atoms - and that it ranges between zero, in the oxidizer and unity in the fuel. The second variable $Y_{c}$ is used to follow the progress of chemical reactions. Among the various possible choices for the progress variable [9], a definition based on the mass fractions of carbon monoxide and dioxide [8] has been retained just for the sake of simplicity: $Y_{c}=0.5 \cdot\left(Y_{\mathrm{CO}}+Y_{\mathrm{CO}_{2}}\right)$. The proposed flame index partitioning framework is independent of the progress variable definition and other definitions can be chosen according to the input fuel or stiffness of chemical source terms. In this respect, it must be emphasized that the above definition of the progress variable has been retained just for the sake of simplicity and consistency with previous analyses performed on the same topic. Tabulated data are described with two hundred points along each direction $Y_{\xi}$ and $Y_{c}$. The tabulated premixed flamelets (TPF) database corresponds to one-dimensional unstrained freely-propagating premixed flames, while the tabulated diffusion flamelets (TDF) database is constructed on the basis of one-dimensional strained counterflow diffusion flames.

Figure 1 gathers temperature, progress variable source term, and $\mathrm{CO}$ mass fraction contours, which are issued from premixed and diffusion flamelet prototypes. In the premixed flame plots, the black lines are associated to flammability limits, beyond which tabulated characteristics are interpolated. In the diffusion flame plots, the black line corresponds to the strained counterflow diffusion flame featuring the smallest strain-rate. For smaller strain-rate values, interpolation with the mixing line is performed.

The premixed flame database is deduced from the detailed solution of freely propagating one-dimensional laminar premixed flames obtained for various values of the mixture fraction $Y_{\xi}$ within the flammability limits [7]. Using the San Diego mechanism [36] and for standard pressure and temperature, the laminar flame speed $S_{L}^{0}$ of a stoichiometric mixture is 0.285 $\mathrm{m} / \mathrm{s}$, while the thermal flame thickness is $\delta_{L}^{0}=0.5 \mathrm{~mm}$. Any flamelet property such as composition, temperature or production rate $\omega_{Y_{c}}$, is represented as a function of the progress variable $Y_{c}$, see the left side of Figure 1. Outside the flammability range, properties are interpolated from solutions obtained at the lean and rich flammability limits and those associated to the pure oxidizer $\left(Y_{\xi}=0.0\right)$ and fuel $\left(Y_{\xi}=1.0\right)$ conditions, i.e., air or fuel at $298 \mathrm{~K}$. Thermophysical and molecular transport properties, e.g., NASA polynomial coefficients, viscosity, conductivity, and molecular weight [9] are subsequently determined from the composition and temperature distributions [39]. In regard to the strained diffusion flamelet database, equations are formulated in a counter-flowing stream geometry and solved in the physical space rather than in the mixture fraction space [12]. Flamelets are generated for several values of 


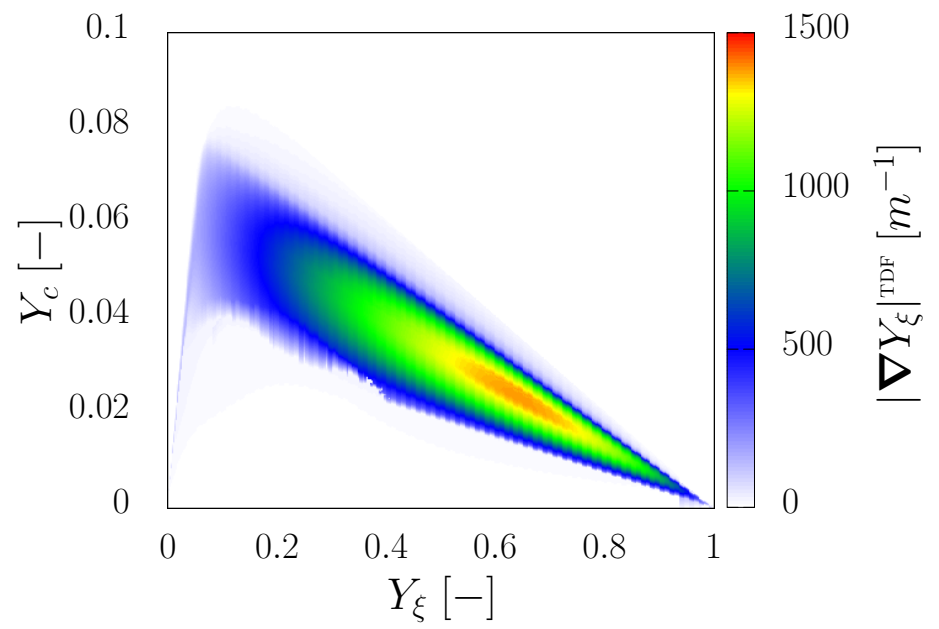

Figure 2.: Distribution of $\left|\nabla Y_{\xi}\right|^{\mathrm{TDF}}$ for diffusion flames of methane and air in standard conditions. Temperature of the inlet streams is $300 \mathrm{~K}$ and the operating pressure is one atm.

the strain-rate $a=\left|u_{O}-u_{F}\right| / d\left[\overline{40]}\right.$, smaller than its value at extinction $a_{q}$. The quantities $u_{O}$ and $u_{F}$ denote the bulk flow velocity of the oxidizer and fuel, respectively, while $d$ refers to the distance between the two nozzles. The solutions obtained in the physical space are then remapped as functions of the mixture fraction. As shown in the top-right plot of Figure 1, in addition to pure mixing, both stable and unstable branches of strained diffusion flame solutions are computed [1341]. The strained diffusion flamelet database is in turn remapped as a function of the progress variable[13]. In this way, flamelet properties of the diffusion flame database will depend on the same set of variables as those retained to parameterize the premixed flamelets, see for instance the $\dot{\omega}_{Y_{c}}$ and the CO plots reported in Figures 1 . Finally, it is noteworthy that, since it is involved in the premixedness index calculation, see Eq. (11), the norm of the mixture fraction gradient $\left|\nabla Y_{\xi}\right|^{\mathrm{TDF}}\left(Y_{\xi}, Y_{c}\right)$ is also tabulated. Its dependence on the mixture fraction and progress variable is depicted in Figure 2 .

\section{Results and discussion}

In this section, the relevance and performance of the proposed flame index are evaluated. To this purpose, three distinct canonical situations featuring equivalence ratio variations are considered: (i) counterflow flames (including premixed, partially-premixed, and diffusion flames), (ii) stabilized triple flames, and (iii) unsteady flame kernel development in nonhomogeneous reactants. The first two cases correspond to steady-state situations, which are considered as standard targeted geometries for multiregime flamelet analyses [8[16]41]. The third case offers the opportunity to evaluate the relevance of the flame index in an unsteady case relevant to the propagation phase that follows the ignition of a non-homogeneous mixture as it may occur in practical situations (e.g., gasoline DI engines).

\subsection{Computational methodology}

For each validation subset, the multiregime flamelet model proposed in the previous section is compared to a reference solution deduced from detailed chemistry (DC) computa- 
tions. As emphasized above, the multiregime model relies on two distinct databases associated to steady and freely-propagating one-dimensional premixed flames and strained diffusion flamelets. These two building blocks correspond to the two fundamental combustion limits of combustion regimes and the computational results obtained with each of the corresponding databases, i.e., tabulated premixed flamelets (TPF) and tabulated diffusion flamelets (TDF), will also be considered as references.

It is noteworthy that some numerical difficulties are avoided by enforcing the premixedness index $\zeta_{\mathrm{PF}}$ to zero for values of the progress variable that are smaller than a given threshold. Similarly, the term $\boldsymbol{n}_{Y_{\xi}} \cdot \boldsymbol{n}_{Y_{c}}$ is removed from Eq. (8) when $\left|\boldsymbol{\nabla} Y_{c}\right|$ falls below a certain limit so as to avoid the singularity. In this case, $\zeta_{\mathrm{PF}}$ depends only on the ratio of mixture fraction gradients magnitudes.

The different modelling approaches have been implemented in the thermophysical module of OpenFOAM-4.0. It solves the following set of conservation equations for mass, momentum, species mass fractions, and enthalpy:

$$
\begin{aligned}
\frac{\partial \rho}{\partial t}+\boldsymbol{\nabla} \cdot(\rho \boldsymbol{u}) & =0 \\
\frac{\partial \rho \boldsymbol{u}}{\partial t}+\boldsymbol{\nabla} \cdot(\rho \boldsymbol{u} \boldsymbol{u})-\boldsymbol{\nabla} \cdot(\mu \boldsymbol{\nabla} \boldsymbol{u}) & =-\boldsymbol{\nabla} p \\
\frac{\partial \rho Y_{i}}{\partial t}+\boldsymbol{\nabla} \cdot\left(\rho \boldsymbol{u} Y_{i}\right)-\boldsymbol{\nabla} \cdot\left(\mathscr{D}_{i} \boldsymbol{\nabla} Y_{i}\right) & =\dot{\omega}_{Y_{i}} \\
\frac{\partial \rho h_{a}}{\partial t}+\boldsymbol{\nabla} \cdot\left(\rho \boldsymbol{u} h_{a}\right)-\boldsymbol{\nabla} \cdot\left(\alpha \boldsymbol{\nabla} h_{a}\right) & =0
\end{aligned}
$$

Consistently with Eqs.(12) and (13), and as aforementioned in Section 2.3, Lewis number effects are not considered (i.e., $\mathscr{D}_{i}=\mathscr{D}=\alpha$ ). The above system is complemented with the perfect gas equation of state $(\mathrm{EoS})$ :

$$
p=\frac{\rho \mathcal{R} T}{\mathcal{M}}, \quad \frac{1}{\mathcal{M}}=\sum_{\alpha=1}^{\alpha=N_{s p}} \frac{Y_{\alpha}}{\mathcal{M}_{\alpha}}
$$

where standard notations have been retained: $\mathcal{R}$ is the universal gas constant, $\mathcal{M}$ is the molecular weight of the multi-component mixture, and $\mathcal{M}_{\alpha}$, the one of the chemical species $\alpha$.

The enthalpy of the mixture is related to temperature through NASA polynomials:

$$
\frac{h_{a}}{R T}=a_{1}+a_{2} \frac{T}{2}+a_{3} \frac{T^{2}}{3}+a_{4} \frac{T^{3}}{4}+a_{5} \frac{T^{4}}{5}+\frac{a_{6}}{T}
$$

where the coefficients $a_{\alpha}$ depend on the composition of the mixture, an expression from which the temperature can be deduced using an iterative procedure [9].

On the one hand, the finite-rate or detailed chemistry computations DC are based on an implementation of the Cantera solver [35] into the thermo-physical module of OpenFOAM. The zero-dimensional constant-pressure reactor is thus used to evaluate the chemical productionconsumption terms that are present in the right-hand-side (RHS) of the chemical species mass fraction transport equations. The relevance of this implementation has been thoroughly verified and some elements of the corresponding validation procedure are provided in Appendix A. On the other hand, the implementation of the flamelet model consists mainly in loading the chemistry tables, from which all properties are read and interpolated. In this case, instead of solving the transport equations for the $N_{s p}$ chemical species present in the detailed chemistry scheme, see Eq. 17, only two species transport equations are solved (i.e., $Y_{\xi}$ and 
$Y_{c}$, as given by Eqs. (12) and (13)). It should be emphasized that the four models, i.e., DC, TPF, TDF, and partitioned tabulated flames (PTF), have been implemented in the same solver and all computations make use of the same discretization strategies and numerical schemes, which are summarized below. For the Laplacian terms, a Gauss discretization with linear interpolation and non-orthogonal correction is used, while a first-order TVD is employed for the convective terms with a value of the LimitedLinear parameter set to 0.5. A secondorder Crank-Nicholson scheme is used for the temporal discretization. Finally, non-reflective boundary conditions are set at the outlet of the computational domain.

\subsection{Laminar counterflow flame configuration}

The relevance of the proposed multiregime combustion or PTF model is first assessed through the consideration of steady-state counterflow partially premixed flames. In this configuration, a premixed methane-air stream is opposed to an oxidizing air stream and a set of reference flames is computed at ambient conditions for several values of the equivalence ratio $\Phi$ in the methane-air mixture. As the equivalence ratio $\Phi$ of the premixed mixture is increased, the flame transitions from a strained premixed flame to rich partially premixed flames until a pure diffusion flame regime is recovered. These limits were previously investigated in the work of Fiorina et al. [8] for methane-air flames. A multiregime combustion model must be able to reconstruct the burning rates obtained for those specific conditions without any additional input of user-defined functions. Thus, a set of reference counterflow flames is defined and computed so as to evaluate the ability of the proposed index to reproduce the burning rates and species distributions in these various conditions. It is noteworthy that, in this one-dimensional geometry, mixture fraction and progress variable gradients are necessarily aligned.

The counterflow flames are computed in a rectangular computational domain, the length of which is not the same for the whole set of computation. It is denoted $d$ in the direction normal to the flame (i.e., $L_{y}=d$, which is the distance between the flow inlets) and it is $3 d$ in the other direction (i.e., $L_{x}=3 d$ ). The corresponding values are provided in flame thickness units in Table 1, with $\delta_{L}^{0}$ denoting the thermal laminar flame thickness at stoichiometry. A symmetry boundary condition is imposed along the line that would connect the center of both inlets so that only half of the flowfield is computed. The remaining boundary conditions include inflow conditions for the reactants, for which the composition, i.e., species mass fraction and temperature) and velocity components are specified. The two inlet streams are set to the same velocity $U=0.3[\mathrm{~m} / \mathrm{s}]$ and inlet temperature of $298[\mathrm{~K}]$. The composition of the oxidizer inlet stream is kept constant and corresponds to air (i.e., $Y_{\mathrm{O}_{2}}=0.233$ and $Y_{\mathrm{N}_{2}}=0.767$ ). A set of flames associated to four distinct values of the equivalence ratios $\Phi$ in the methane/air inlet is considered for different values of the strain rate $a$. The corresponding values are presented in Table 1. The corresponding conditions are associated to a diluted premixed flame $(\Phi=1.0)$ stabilized against air, the onset of a rich partially premixed flame $(\Phi=1.8)$, a rich partially premixed flame $(\Phi=3.0)$, and the onset of a pure diffusion flame $(\Phi=5.0)$. It is noteworthy that these conditions are associated to a wide range of composition variations for which the heat and mass transfer across equivalence ratio iso-surfaces may play an important role.

The following subsections describe the flame structure of these counterflow flames with a focus placed on the analysis and validation of the proposed partitioning index, so the influence of both the equivalence ratio and strain-rate is discussed. From previous works on multiregime flamelet modelling [8]16/41], it is known that major species like $\mathrm{CO}_{2}$ or $\mathrm{H}_{2} \mathrm{O}$ are highly temperature-dependent and their predictions are ultimately related to temperature. This is in contrast with the prediction of intermediate species or unburnt hydrocarbons molecules, which are more challenging as their formation is more sensitive to the radical pool. Therefore, 
Table 1.: Parameters retained to perform the numerical simulations of the counter-flow flames (the value of $S_{L}^{0}$ corresponds to the laminar propagation velocity obtained at stoichiometry).

\begin{tabular}{cc|cccc}
$\Phi$ & $a\left[\mathrm{~s}^{-1}\right]$ & $d / \delta_{L}^{0}$ & $\delta_{L}^{0} / \Delta y$ & $\Delta x / \Delta y$ & $U / S_{L}^{0}$ \\
\hline \hline 1.0 & 279 & 4.33 & 50 & 2 & 1.054 \\
1.8 & 395 & 3.06 & 50 & 2 & 1.054 \\
3.0 & 173 & 6.94 & 50 & 2 & 1.054 \\
3.0 & 296 & 4.07 & 50 & 2 & 1.054 \\
3.0 & 381 & 3.16 & 50 & 2 & 1.054 \\
5.0 & 356 & 3.39 & 50 & 2 & 1.054
\end{tabular}

the next subsections are focused on the study of the evolution of temperature and $\mathrm{CO}$ mass fraction, together with those of two other radicals $\left(\mathrm{OH}\right.$ and $\left.\mathrm{CH}_{3}\right)$, across the one-dimensional partially premixed flames.

\subsubsection{Influence of equivalence ratio and strain-rate}

Results of counterflow flames obtained for various equivalence ratios at the fuel/air inlet stream are first presented for a given value of the strain-rate $a$ close to the extinction point. The temperature and $\mathrm{CO}$ mass fraction distributions along the domain centerline are plotted in Fig. 3. The results show that the solution obtained with the proposed flame index lies between those issued from tabulated premixed and tabulated diffusion flamelets. In general, the PTF model leads to a better level of agreement with the reference DC solution in comparison with the solution issued from the sole consideration of either tabulated premixed or tabulated diffusion flamelets.

For an equivalence ratio $\Phi=1.0$, the flame is fully premixed and the result from tabulated diffusion flamelets (TDF) differs significantly from the DC solution. In this case, the flame index recovers the premixed flamelets solution and the correlation with the detailed chemistry (DC) solution is satisfactory. By increasing the equivalence ratio up to $\Phi=1.8$, molecular diffusion fluxes across equivalence ratio surfaces come into play and the solution obtained with tabulated diffusion flamelets gets closer to the reference DC solution, while the consideration of tabulated premixed flamelets (TPF) leads to larger differences with the reference solution. The proposed flame index captures fairly well these partial premixing effects and leads to a better correlation with the reference temperature and $\mathrm{CO}$ concentration compared to those obtained with either TPF or TDF. As the inlet equivalence ratio value is further increased up to $\Phi=3.0$ and $\Phi=5.0$, a double-flame structure that evolves into a diffusion flame is formed. These partially premixed flames feature a rich premixed flame followed by a diffusion flame front between the unburnt gases and the opposite air stream in the vicinity of the stagnation point. Diffusion flamelets are found able to predict these flow conditions, while premixed flamelets are no longer valid to reproduce the burning rates and flame structure, especially for rich conditions. This trend is well captured by the proposed multiregime model, which balances out the contribution of the premixed burning at diffusion-dominated reacting conditions. We conclude that the proposed flame index leads to better temperature predictions than those obtained with diffusion flamelets alone, although this is not the case for the $\mathrm{CO}$ concentration as it is over-predicted as a consequence of the additional contribution of premixed flamelets.

As shown in Fig. 4, a similar behavior is observed for methyl $\mathrm{CH}_{3}$ and hydroxyl $\mathrm{OH}$ radicals. The multiregime model is found able to reproduce the distributions of these two radicals for the different flow conditions. The agreement obtained with DC computations is remark- 

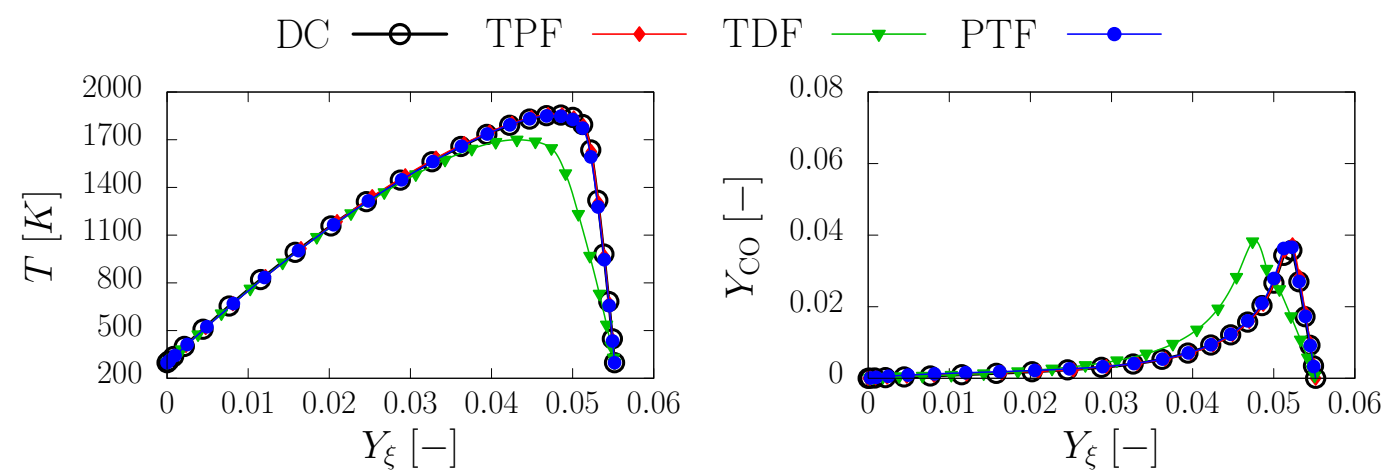

(a) $\Phi=1.0, a=279 \mathrm{~s}^{-1}$
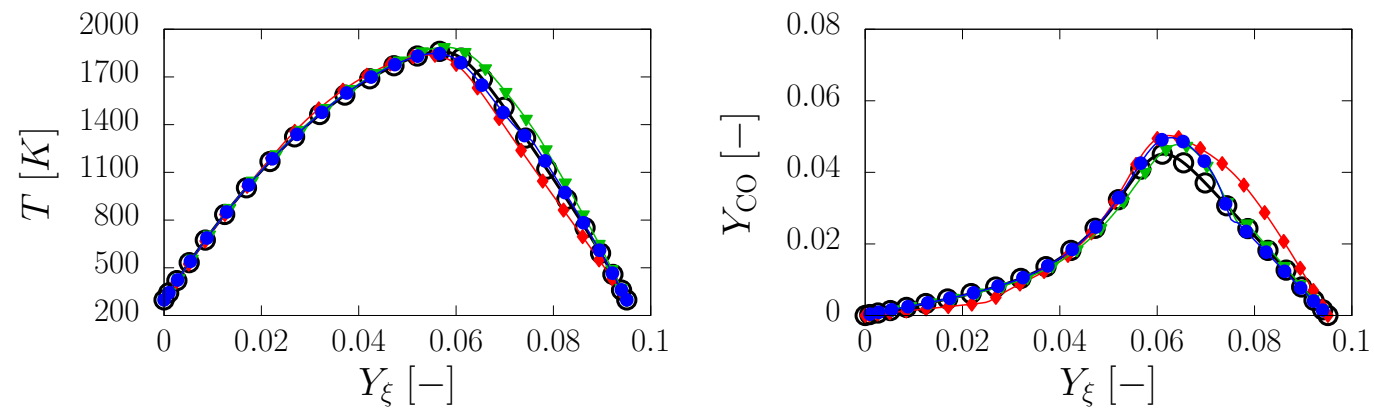

(b) $\Phi=1.8, a=395 \mathrm{~s}^{-1}$
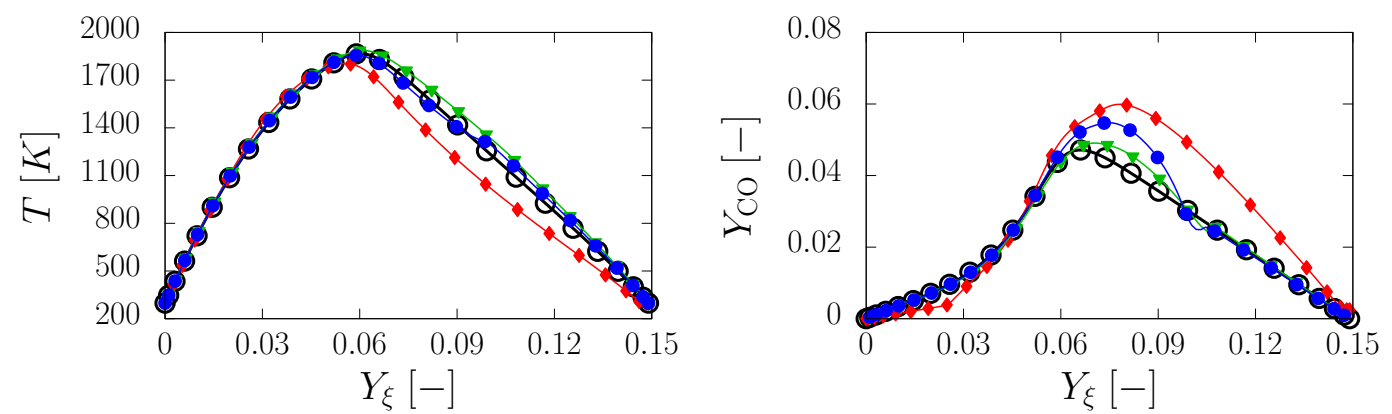

(c) $\Phi=3.0, a=381 \mathrm{~s}^{-1}$
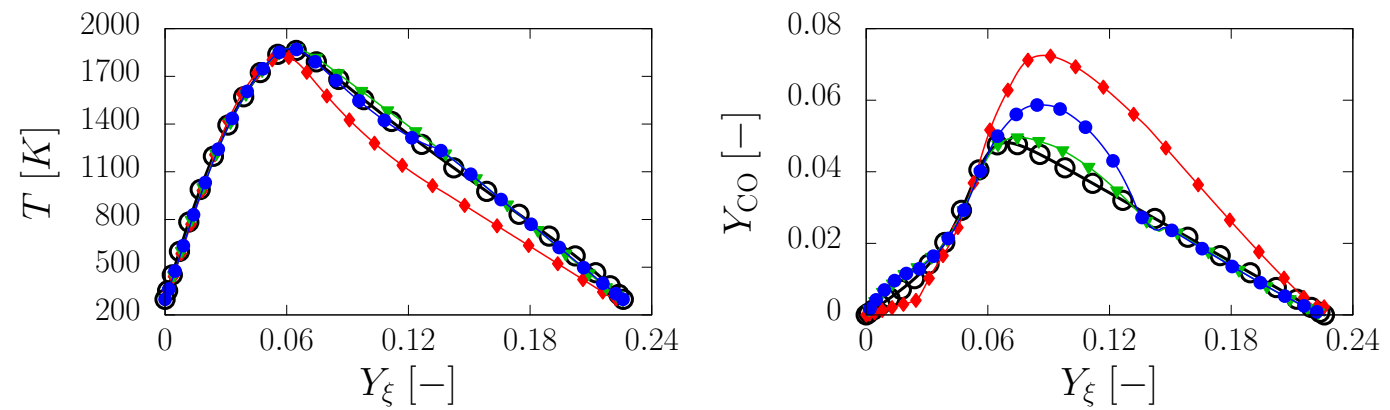

(d) $\Phi=5.0, a=356 \mathrm{~s}^{-1}$

Figure 3.: Profiles of temperature $T$ (left) and mass fraction of $\mathrm{CO}$ (right) along the centerline of premixed counter-flow flames obtained close to the extinction for different inlet equivalence ratio $\Phi$. 
able for the $\mathrm{OH}$ profiles, while the $\mathrm{CH}_{3}$ concentration is slightly under-predicted, due to the premixed flamelets contribution. This is in agreement with the results obtained for the $\mathrm{CO}$ concentration.

The influence of the strain-rate on the prediction capabilities of the proposed flame index is now investigated. For this purpose, the results obtained in the case of the rich partially premixed flame $(\Phi=3.0)$ for three distinct values of the strain-rate, see Table 1 , are analyzed. The distributions of the temperature and burning rate of the progress variable are displayed in Fig. 5. The influence of the strain-rate on the flame temperature and burning rates is clearly evidenced. For low strain-rate conditions, the partially premixed flame can be represented by laminar one-dimensional tabulated premixed flamelets, while for larger values of the strainrate, it is better represented when using a tabulated diffusion flame representation.

Finally, despite the burning rates and temperature distributions obtained with the proposed flame partitioning are in good agreement with the detailed chemistry computations, it must be recognized that the flame structure is not fully recovered. Indeed, from the mass fraction profiles of some given chemical species and radicals that are obtained for an intermediate value of the strain-rate, as shown in Fig. 6, it can be seen that the TPF contribution issued from the flame index partitioning leads to an over-prediction of the overall mass fraction of $\mathrm{CO}$ on the rich side of the flame. In fact, for the lowest strain-rate value, i.e., $a=173 \mathrm{~s}^{-1}$, one can notice that the $\mathrm{CO}$ mass fraction tends to be over-predicted within the mixture fraction range $Y_{\xi} \in[0.07,0.12]$. The corresponding range, as well as the associated deviations from the reference solution, is however reduced as the value of the weighting variable $\zeta_{\mathrm{PF}}$ becomes smaller, which corresponds to a strain-rate increase. At this level, it is noteworthy that the prediction of certain species may be improved by considering other progress variable definitions.

\subsubsection{Flame index partitioning analysis}

The results presented in the previous subsection have not only shown the ability of the proposed flame index to adapt to different flame regimes but also its capacity to provide weighting factors that reproduce the burning rates of the progress variable from a combination of premixed and diffusion flamelets. The weighting factor distributions obtained for the selected conditions are displayed in Fig. 7. In order to compare the distribution of the present premixedness index with more standard expressions provided in the literature, such as those given by Eqs. (5) and (6), Fig. 7 shows different index proposals along with the rate of reaction of the progress variable. It is noteworthy that the proposed flame index is used only for non-negligible values of the progress variable $Y_{c}$, otherwise the properties are taken from tabulated diffusion flamelets, which gives a better representation of the non-reactive molecular mixing processes.

The obtained distribution of the flame index is typical of counterflow flame structures. For the strained premixed flame $(\Phi=1)$, the flame index $\zeta_{\mathrm{PF}}$ is distributed over the region where the premixed flame is stabilized. As the flame becomes partially premixed by increasing $\Phi$, the profile of the premixedness index $\zeta_{\mathrm{PF}}$ widens in the mixture fraction space and the corresponding peak value is reduced. This evolution leads to a larger contribution of diffusiondominated burning featured by diffusive fluxes across equivalence ratio iso-surfaces; it results also in an improvement of the burning rates and transport properties estimation with respect to the results obtained with tabulated premixed flamelets. The proposed approach leads also to a zero weighting factor in the whole computational domain for the case of a diffusion flame. The value of $\zeta_{\mathrm{PF}}$ reduces to zero in rich conditions, which are better represented by diffusion flamelets, as stated above in Section 3.2.1. The influence of the strain-rate on the flame index is also shown for a rich partially premixed flame with $\Phi=3.0$. As the increase in strain- 

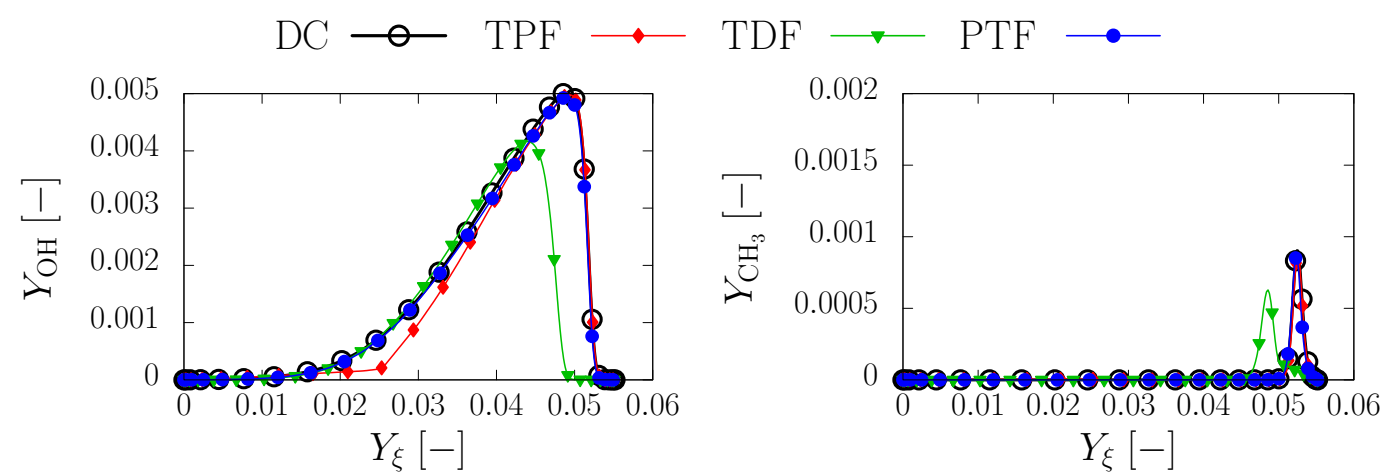

(a) $\Phi=1.0, a=279 \mathrm{~s}^{-1}$
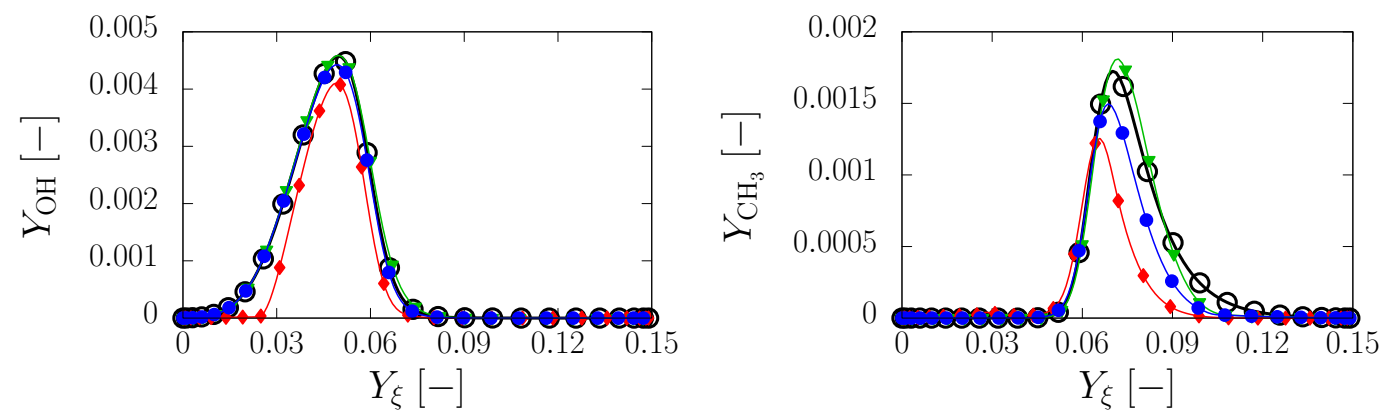

(b) $\Phi=3.0, a=381 \mathrm{~s}^{-1}$
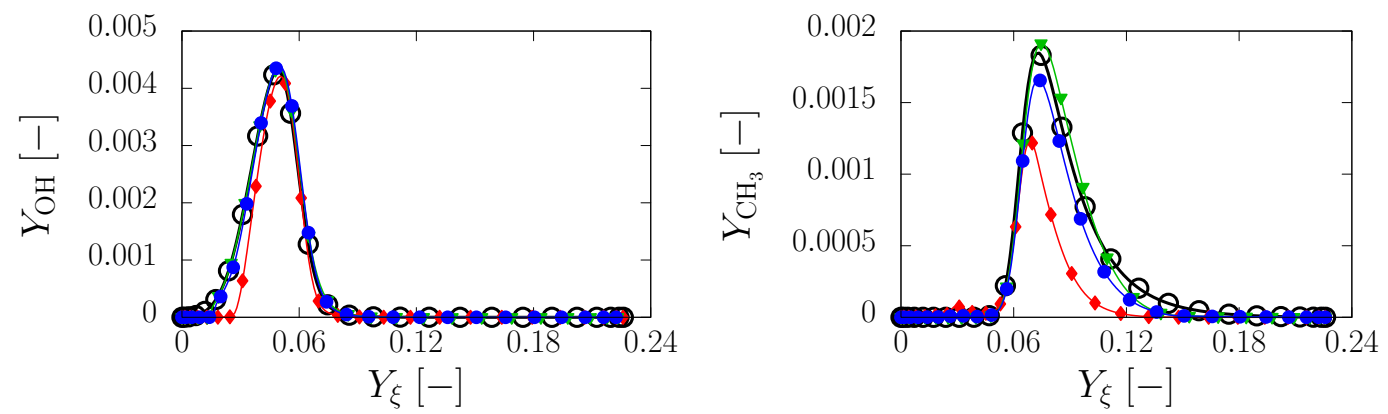

(c) $\Phi=5.0, a=356 \mathrm{~s}^{-1}$

Figure 4.: Profiles of mass fraction of $\mathrm{OH}$ (left) and $\mathrm{CH}_{3}$ (right) along the centerline of counter-flow flames obtained close to the extinction for different inlet equivalence ratio $\Phi$. 

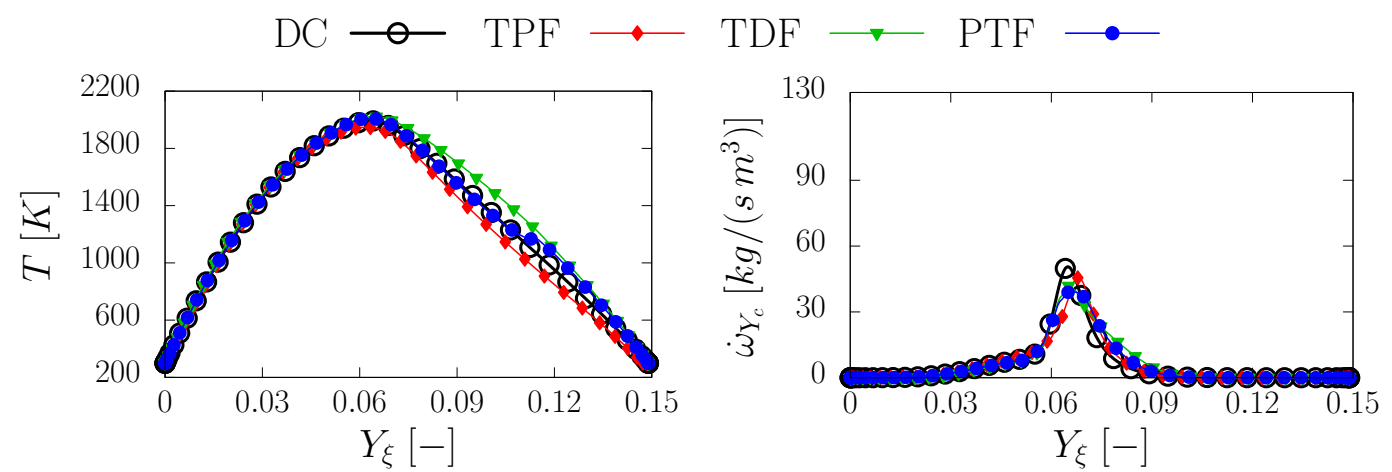

(a) $a=173 \mathrm{~s}^{-1}$
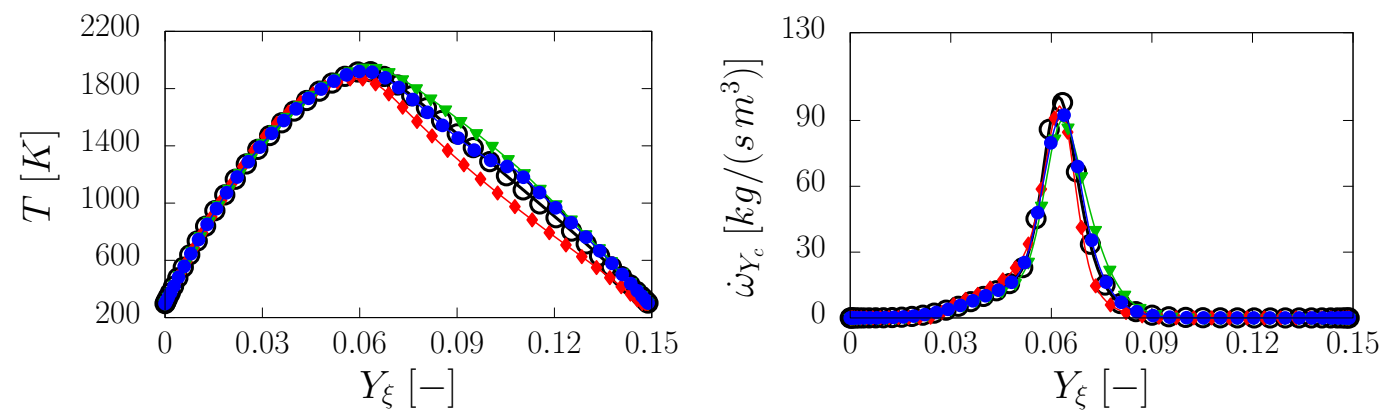

(b) $a=296 \mathrm{~s}^{-1}$
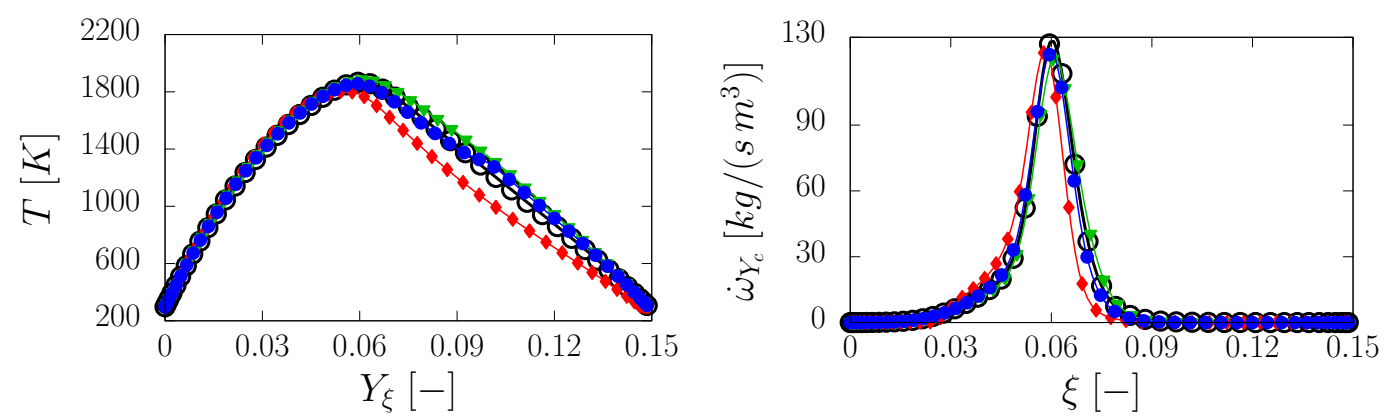

(c) $a=381 \mathrm{~s}^{-1}$

Figure 5.: Profiles of temperature $T$ (left) and progress variable production rate $\dot{\omega}_{Y_{c}}$ (right) along the centerline of premixed counter-flow flames obtained for $\Phi=3.0$ and different strain-rate values. 

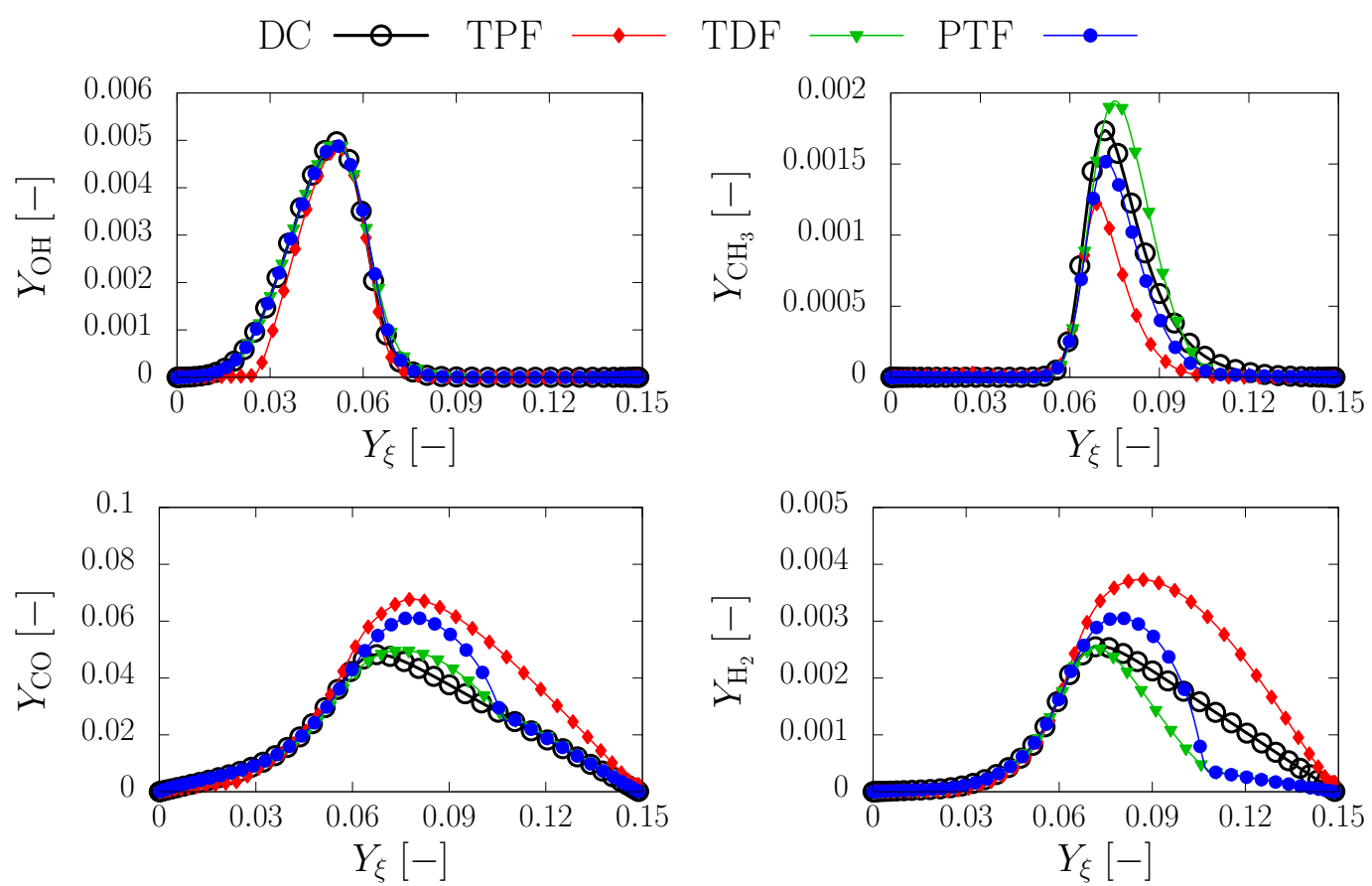

Figure 6.: Profiles of several species mass fractions along the centerline of premixed counterflow flames obtained for $\Phi=3.0$ at $a=381 \mathrm{~s}^{-1}$

rate displaces the flame front towards the stagnation plane, the rich premixed leading edge gets leaner as a result of dilution with the incoming air. This leads to higher peak values of the index for smaller values of the mixture fraction when the strain-rate increases. The peak values are also slightly reduced, a feature associated with a strong diffusive burning at larger strain-rates. Finally, it is worth noting that the generalized premixedness index shifts abruptly from premixed to diffusion combustion within the reactive layer and seems rather insensitive to strain-rate variations, which explains its ability to change both the burning rate and flame structure.

\subsection{Triple flame configuration}

The second test case under investigation is the two-dimensional triple flame, which is considered as an archetypal case of partially premixed combustion. There have been many studies dedicated to the characterization of triple flames in the literature [7]42], where the effects of partial premixing, heat release and autoignition have been thoroughly analysed. Therefore, this test case has been used frequently for the analysis of combustion models in partiallypremixed conditions [7|15|16].

From the stoichiometric point located at the leading edge, a rich partially premixed flame develops towards the fuel side, while a lean partially premixed flame is established towards the oxidizer stream. The specific point where the branches merge is called the triple point [42]43]. Downstream of the partially premixed flame, chemical reactions leads to the formation of a trailing post-diffusion flame.

The mixture fraction distribution in the incoming stream of unburnt reactants is one of the main parameters controlling the characteristics of the triple flame [7]. The equivalence ratio distribution drives the burning rates obtained at the leading edge, influencing the position of the reactive layer, and the resulting combustion regime. Thus, triple flames introduce a 


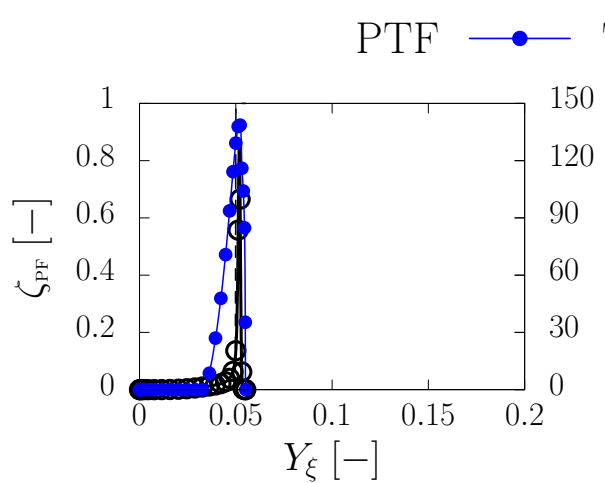

(a) $\Phi=1.0, a=279 \mathrm{~s}^{-1}$

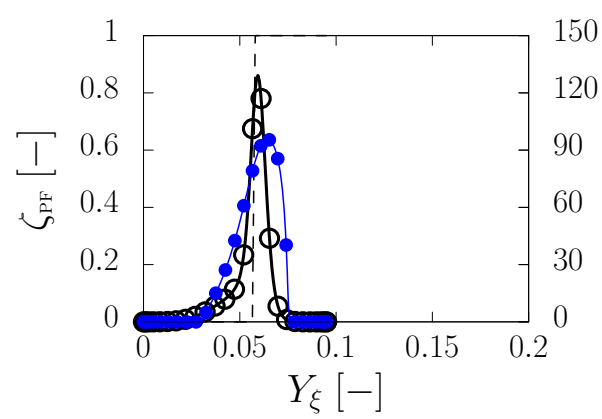

(c) $\Phi=1.8, a=395 \mathrm{~s}^{-1}$

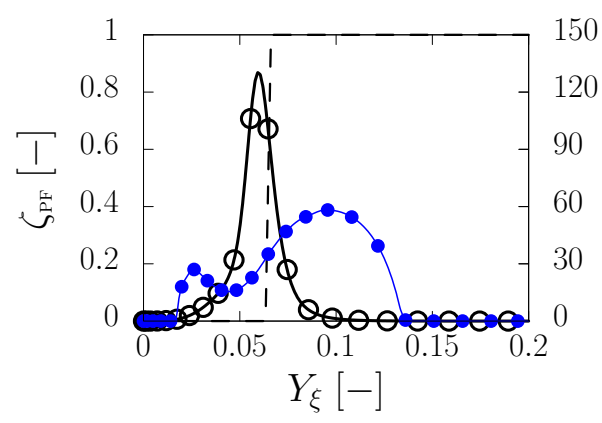

(e) $\Phi=5.0, a=356 \mathrm{~s}^{-1}$
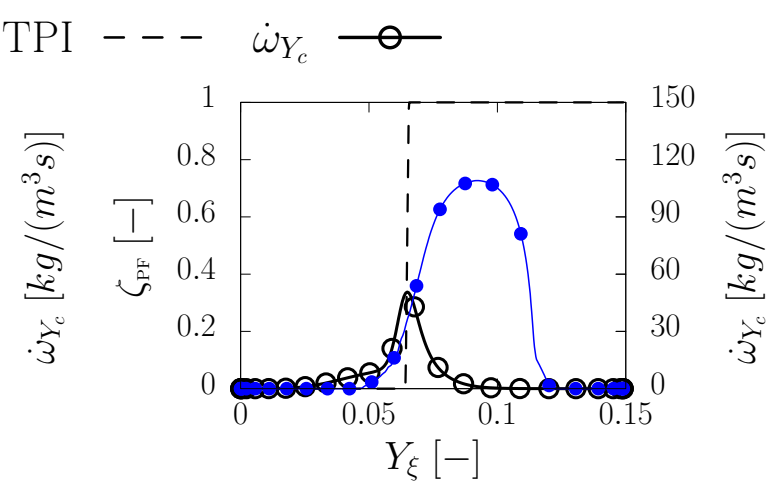

(b) $\Phi=3.0, a=173 \mathrm{~s}^{-1}$

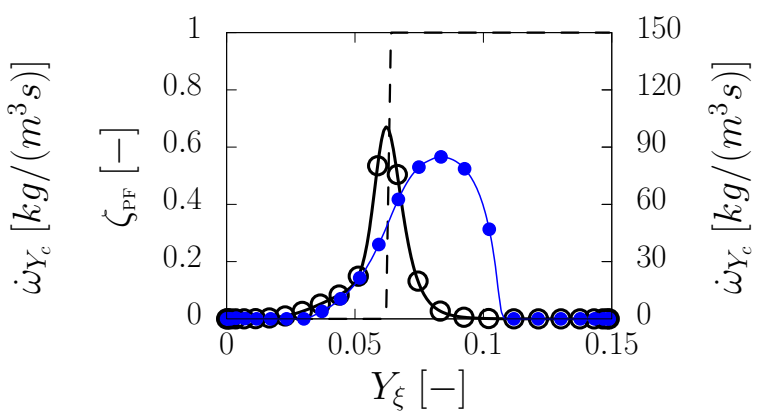

(d) $\Phi=3.0, a=296 \mathrm{~s}^{-1}$

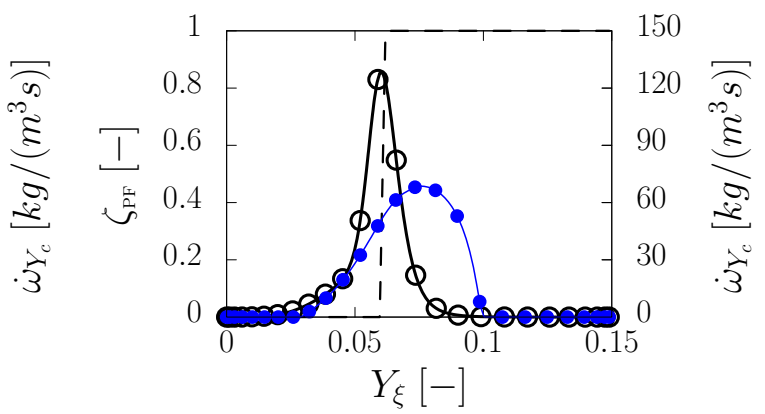

(f) $\Phi=3.0, a=381 \mathrm{~s}^{-1}$

Figure 7.: Flame premixedness index profiles along the centerline of premixed counterflow flames obtained for different equivalence ratios (left) and strain-rates (right).

stronger coupling between the mixture fraction and progress variable fields when compared to the case of counterflow flames such as those considered in the previous section of this study. Moreover, in contrast to the previous test case, mixture fraction and progress variable gradients may be misaligned. The development of molecular diffusion fluxes of mass and energy in two-dimension have a large impact on the burning rates, and therefore influences the stabilization point and the flame topology itself. From previous studies of triple flames performed with flamelet methods [7|15[44], it is known that, in conditions associated to moderate mixture fraction gradients, tabulated premixed flamelets lead to better predictions of the flame burning rates [7], while triple flames developing in high mixture fraction gradients are better represented when using tabulated diffusion flamelets [41]. 
In the present study, three different inlet conditions are defined according to Eq. 21]:

$$
Y_{\xi}(0, y)=\frac{1}{2}\left(1.0-\tanh \frac{y}{b}\right)
$$

where $b$ denotes a typical mixing layer thickness, see Fig. 8. These three distinct mixture fraction profiles are used to analyze the effect of the mixture fraction gradient on the triple flame propagation and topology. The corresponding three different conditions are hereafter referred to as cases A, B, and C. It is noteworthy that situations associated to moderate mixture fraction gradients (similar to the present case A) correspond to standard benchmarks in the literature because they clearly exhibit both premixed and diffusion modes of combustion [15, 16].

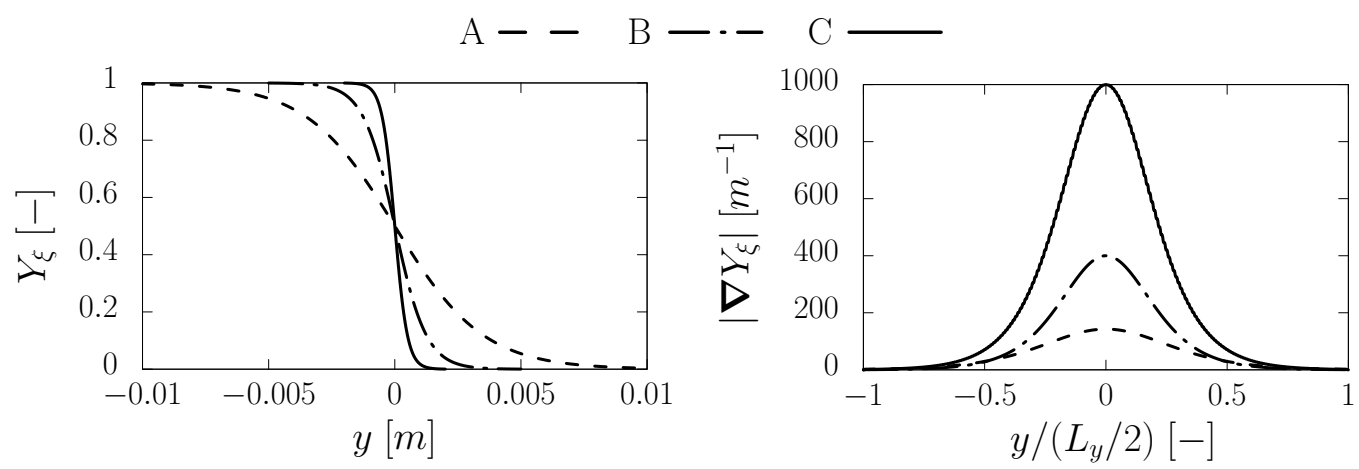

Figure 8.: Mixture fraction profile $Y_{\xi}(0, y)$ (left) and associated magnitude of its gradient (right) at the inlet of the computational domain

The rectangular computational domain features an inlet and outlet boundary conditions on its left and right sides, respectively. Symmetry conditions are applied at the top and bottom. The parameters associated to each simulated case are gathered in Table 2 . The measured flame propagation speed $S_{\mathrm{TF}}$ of the triple flame corresponds to the normal velocity component imposed as the left boundary condition, so that a steady triple flame is obtained within the computational domain.

Table 2.: Parameters relevant to the numerical simulations of triple flames. The characteristic mixing length $\delta_{m}$ is estimated from $\left(Y_{\xi}^{\max }-Y_{\xi}^{\min }\right) /\left|\nabla Y_{\xi}\right|^{\max }$ with the maximum of the mixture fraction gradient $\left|\nabla Y_{\xi}\right|^{\max }$ taken either at the inlet $\left(\delta_{m}^{i n}\right)$ or at the leading edge $\left(\delta_{m}^{L E}\right)$.

\begin{tabular}{c|ccccccc} 
& $b \cdot 10^{3}$ & $L_{y}[\mathrm{~mm}]$ & $L_{x}[\mathrm{~mm}]$ & $\delta_{L}^{0} / d y$ & $\delta_{m}^{i n} / \delta_{L}^{0}$ & $\delta_{m}^{L E} / \delta^{0}$ & $S_{\mathrm{TF}} / S_{L}^{0}$ \\
\hline \hline case A & 3.0 & 20.0 & 40.0 & 20.0 & 14.0 & 12.45 & 2.11 \\
case B & 1.25 & 10.0 & 20.0 & 25.0 & 5.0 & 4.90 & 1.58 \\
case C & 0.5 & 4.0 & 10.0 & 50.0 & 2.0 & 2.64 & 0.88
\end{tabular}

The resulting triple flame structures are displayed in Figure 9 . The corresponding figure reports mixture fraction and normalized progress variable iso-lines superimposed on the temperature field. The mixture fraction iso-lines are represented by blue lines, while progress variable iso-lines do appear in red. The effect of the mixture fraction inlet profile on the triple flame stabilization is clearly put into evidence. Indeed, the triple flame exhibits a reduced propagation speed $S_{\mathrm{TF}}$ as the value of the mixture fraction gradient magnitude is increased. This comes together with an increased asymmetry of the flame structure with respect to the stoichiometric mixture fraction iso-line. This set of plots also displays significant differences 
in terms of mixture fraction and progress variable iso-lines evolution. As long as the equivalence ratio gradient remains rather moderate at the inlet, the mixture fraction iso-lines are only slightly deflected across the flame front with all progress variable iso-lines merging at the triple point. As the inlet mixture fraction gradient is increased, the molecular diffusion transport between mixture fraction iso-lines becomes more important and these iso-lines tend to get apart from each other, which is in agreement with previous analyses [7]. This behaviour may explain why the use of tabulated premixed flamelets and tabulated diffusion flamelets is appropriate for small and large mixture fraction gradients, respectively. It should be emphasized that premixed flame propagation also takes place for the highest mixture fraction gradient case. The misalignement between the mixture fraction and progress variable gradients is depicted on the right side of Figure 9. These vectors are plotted over an iso-line of normalized progress variable. As expected, the misalignment between the two vectors is very important in the direct vicinity of the leading edge, whereas the two vectors are almost aligned on the lean and rich branches of the triple flame. It is also worth noting that, for case $\mathrm{C}$, the flame region is thicker and mixture fraction variations may occur while chemical reactions proceed. This is in contrast to situations associated with moderate mixture fraction gradient at the inlet. In that case, it seems that mixture fraction variations indeed remain quite negligible over length scales that are typical of the flame thickness. Therefore, the influence of molecular diffusion between mixture fraction iso-lines can be neglected and the edge of the partially premixed flame front can be described as a collection of premixed flamelets. These aspects are discussed in the next subsections with a focus placed on the analysis of the proposed index partitioning to predict these complex burning conditions.

\subsubsection{A priori analysis}

A flame partitioning index should take into account the most appropriate flame properties of premixed and diffusion combustion regimes. In order to assess the suitability of the proposed index, an a priori comparison is first performed based on the DC computational results. The DC solution fields are read by the flamelet solver and a solution is generated for each of the models, maintaining the computed scalar (i.e., $Y_{\xi}$ and $Y_{c}$ ) and velocity fields unchanged. This allows a direct identification of the differences that exist between the models.

The distributions of the progress variable burning rates obtained with the different approaches are compared with the DC solutions in Fig. 10. It is noteworthy that the computaional domain is not the same for all simulated cases. For case A (i.e., low mixture fraction gradient), its characteristic size is doubled with respect to case $\mathrm{B}$, which is associated to a moderate mixture fraction gradient $\left|\nabla Y_{\xi}\right|$ at the inlet. It is five times larger for case A compared to case $\mathrm{C}$, which corresponds to the case of the large mixture fraction gradient. Taking this into account, as $\left|\nabla Y_{\xi}\right|$ increases, the lean branch of the triple flame shortens, while its rich branch elongates and becomes thicker. By taking a closer look at the computational results, it is noticeable that the TDF model leads to a thinner and sharper lean edge with a thicker rich branch. On the other hand, the TPF model improves the result for the first two conditions (cases A and B), while it results in a thin rich branch for the high mixture fraction gradient case (case C). The computational results obtained with the proposed index are similar to those issued from the TPF model in case A. Nevertheless, as $\left|\nabla Y_{\xi}\right|$ is increased, the present model, i.e., the PTF model, does recover some characteristics of the TDF database. Results issued from a blending procedure based on the Takeno premixedness index (TPI) are also included as references.

A more quantitative comparison is provided in Fig. 11, which reports temperature profiles plotted versus mixture fraction conditioned to three values of the normalized progress variable, that is $c=\{0.3,0.6,0.9\}$. For rich conditions (i.e., values of $Y_{\xi}$ larger than 0.055), the 


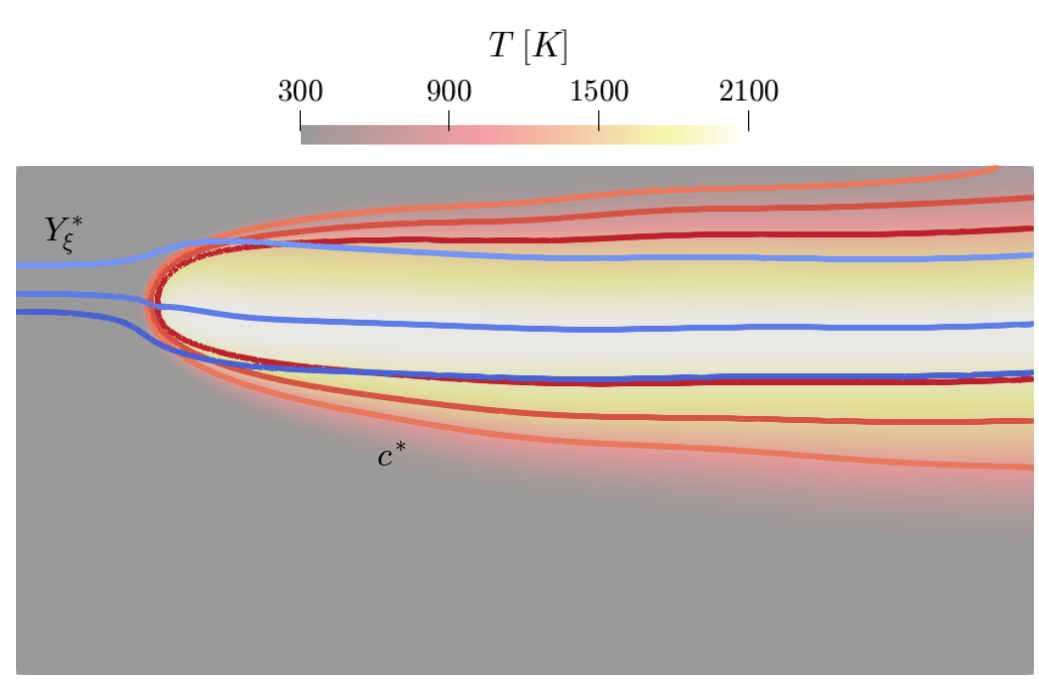

$$
\dot{\omega}_{Y c}\left[k g / m^{3} s\right]
$$

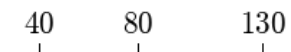

(a) Low gradient
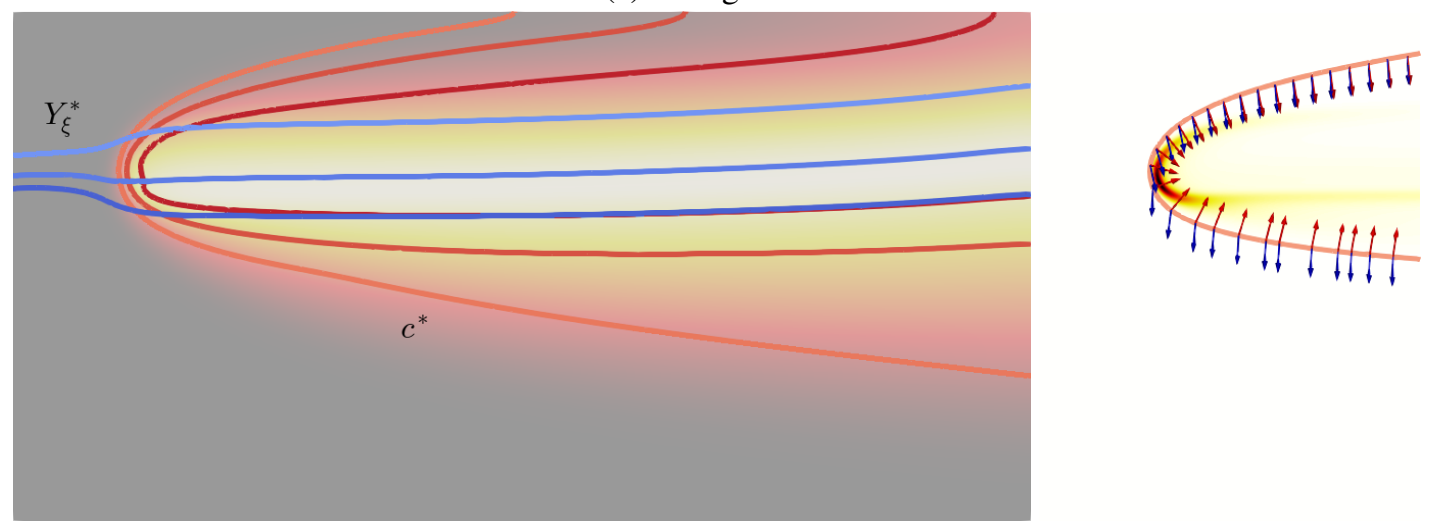

(b) Moderate gradient
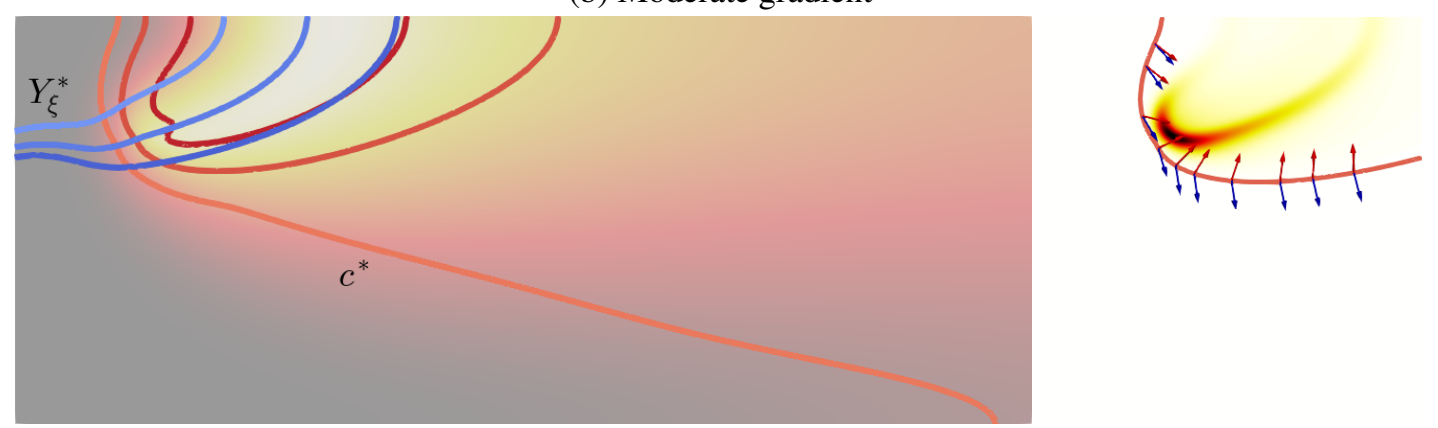

(c) High gradient

Figure 9.: Mixture fraction $Y_{\xi}^{*}=\{0.04,0.055,0.07\}$ and progress variable $c^{*}=\{0.3,0.6,0.9\}$ iso-lines superimposed on the temperature field issued from the DC computations of the triple flame (left) and iso-line $c^{*}=0.5$ plotted together with vectors $\nabla Y_{\xi}$ (blue) and $\boldsymbol{\nabla} Y_{c}$ (red) superimposed on the source term field (right). 

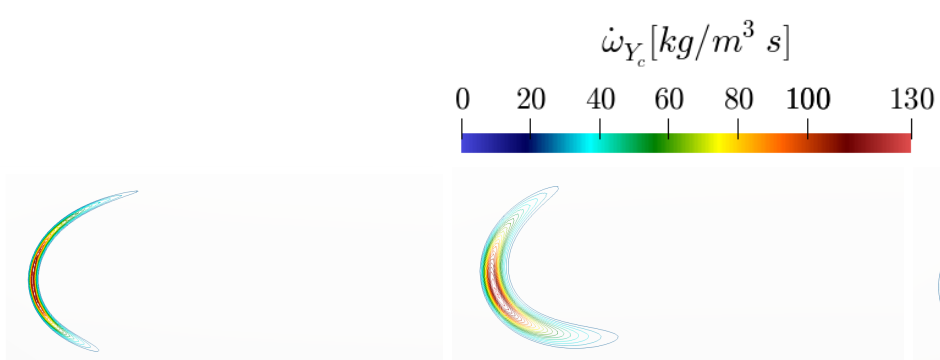

(a) DC
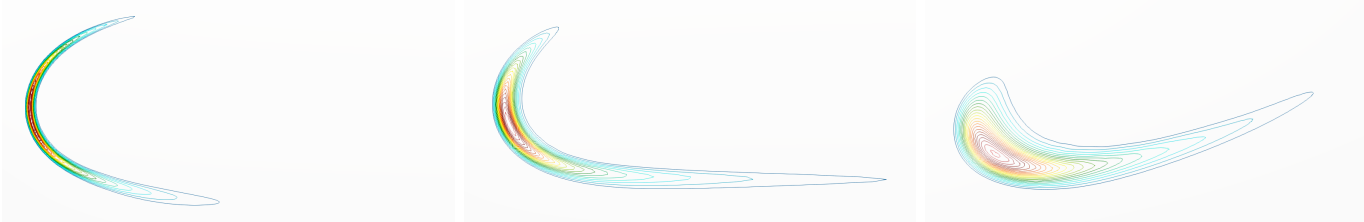

(b) TPF
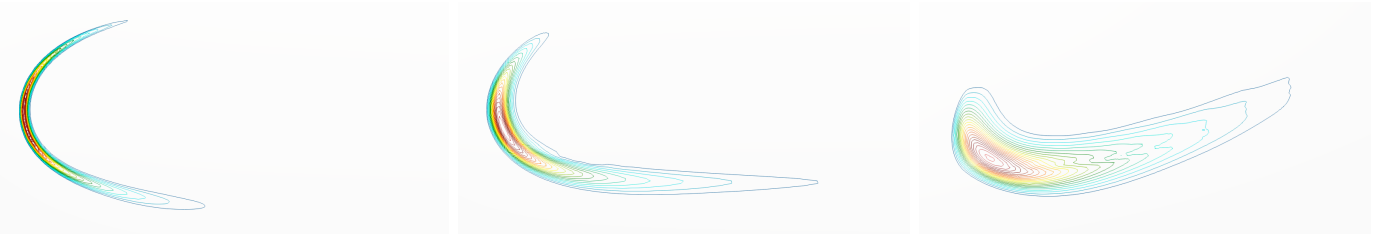

(c) PTF
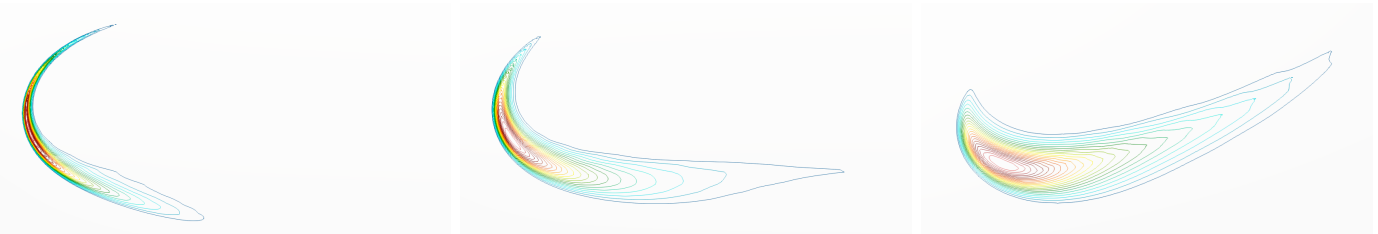

(d) TDF
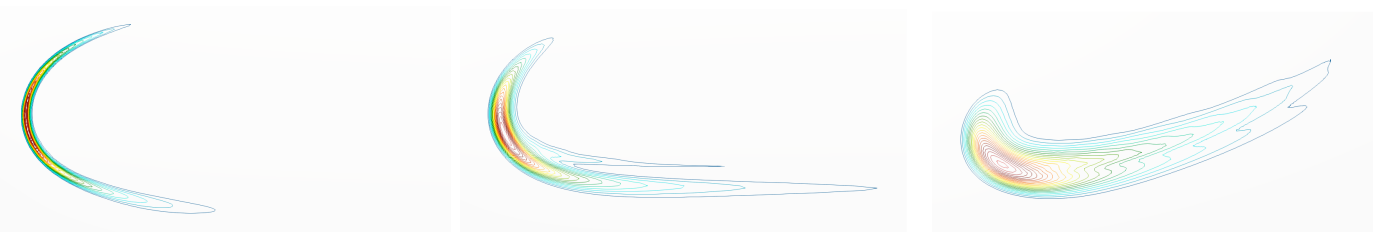

(e) TPI

Figure 10.: Progress variable source term contours for the low (left), moderate (middle), and high (right) inlet mixture fraction gradient cases. 
DC results lie between the TPF and the TDF solutions. From a general point of view, the temperature issued from the reference DC simulation is closer to the TPF solution for moderate values of the progress variable $c$ and it approaches the TDF solution as chemical reaction proceeds towards equilibrium (i.e., $c=0.9$ ). The same conclusion holds whatever the value of the inlet mixture fraction gradient (i.e. from case $\mathrm{A}$ to case $\mathrm{C}$ ). It is also noteworthy that the TPF solution provides a better approximation of the DC results for case A (i.e., moderate mixture fraction gradient at the inlet). Figure 11 also shows that the proposed index (i.e., PTF) is able to predict the temperature levels on the lean side of the leading edge of the triple flame, see the results obtained for $c=0.3$. For higher values of the normalized progress variable $c$, a satisfactory level of agreement is also obtained between the DC reference solution and the PTF results, thanks to the TDF contribution. Even though the temperature appears to be slightly underestimated for these conditions, the PTF results remain better correlated with the DC solutions than those obtained with the sole consideration of tabulated premixed flamelets model). As it was shown above in Fig. 10 the results obtained with the TPI model follows the same trends as those observed with the TPF model and does not exhibit any special sensitivity to the mixture fraction length scale that characterizes the inlet boundary. Due to these limitations, the TPI model is not considered in the forthcoming parts of the analysis.

\subsubsection{A posteriori analysis}

In contrast to the a priori analysis, the a posteriori analysis requires the computation of the reacting flow field presented above with each of the combustion models (i.e., TPF, TDF, and PTF) until a steady-state solution is obtained. Then, the corresponding results are compared to the DC solution. For the sake of conciseness, this detailed comparison between the various model behaviours is focused on case B.

From a fundamental point of view, the position and stabilization point of the triple flame is determined by the propagation speed of the flame leading edge across the mixture fraction and velocity fields. Thus, the ability to predict the corresponding location may be thought as a measure of how a model reproduces the multiregime combustion processes. This can be analyzed through the distribution of the progress variable source term contours that are reported in Fig. 12. The corresponding plots show the influence of the computed burning speeds on the stabilization point of the triple flame. As the leading edge of the triple flame is predominantly premixed, the TPF model seems better suited to capture the evolution of the flame front. The TDF model significantly under-estimates the propagation velocity and the flame stabilizes further downstream. The proposed flame index recovers this leading premixed-dominated flame displayed on the right side of this figure using iso-lines of the progress variable source term $\dot{\omega}_{Y_{c}}$. The DC solution shows that burning rates across mixture fraction surfaces are typical of diffusion flames. Finally, the PTF model offers a good compromise between both tabulated flamelets models (i.e., TPF and TDF), as it predicts the burning rate of a thin premixed flame front at the leading edge, while a diffusive reactive layer develops further downstream. Nonetheless, the PTF model tends to overestimate the thickness of the post-diffusion flame, which is an outcome of the TPF contribution.

Figure 13 displays the profiles of the progress variable source term $\dot{\omega}_{Y_{c}}$ and $\mathrm{CO}$ mass fraction plotted versus the normalized progress variable for three distinct values of the mixture fraction. These plots show that the flame burning rates $\dot{\omega}_{Y_{c}}$ are better predicted with tabulated premixed flamelets (TPF) for the lean, stoichiometric, and rich sides of the flame. This is in contrast with the CO profiles, for which the TPF model seems to be appropriate only for lean and stoichiometric conditions, while the TDF model performs better for rich conditions. The plots show how the PTF model is able to adapt to these burning conditions. It is clear from 

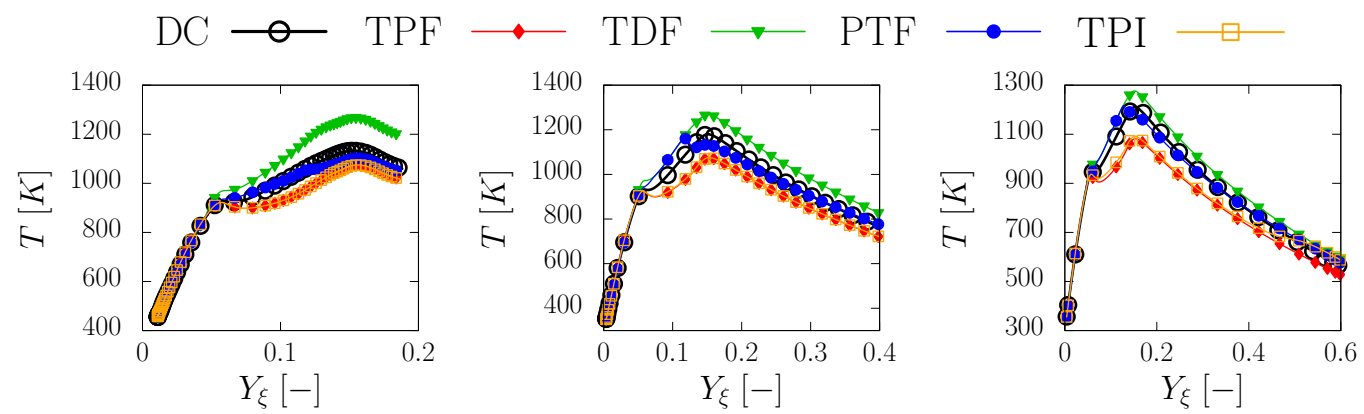

(a) $c=0.3$
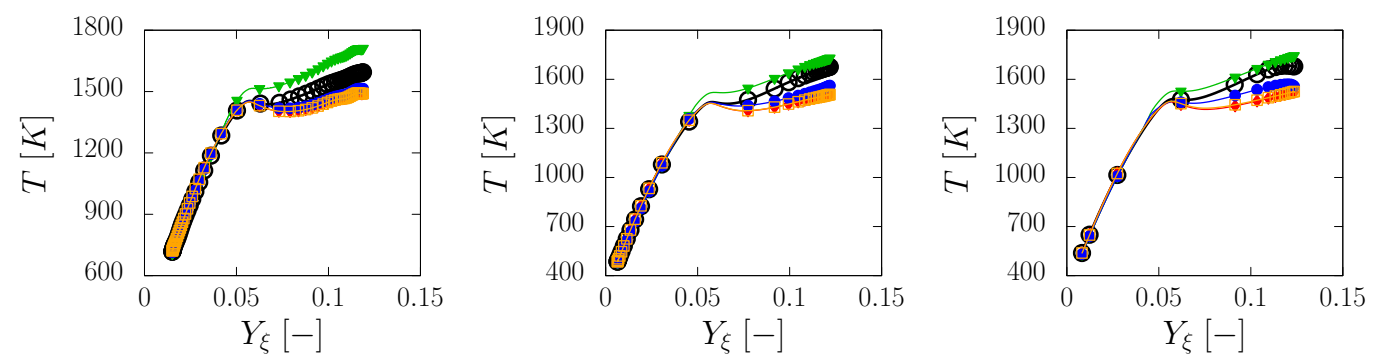

(b) $c=0.6$
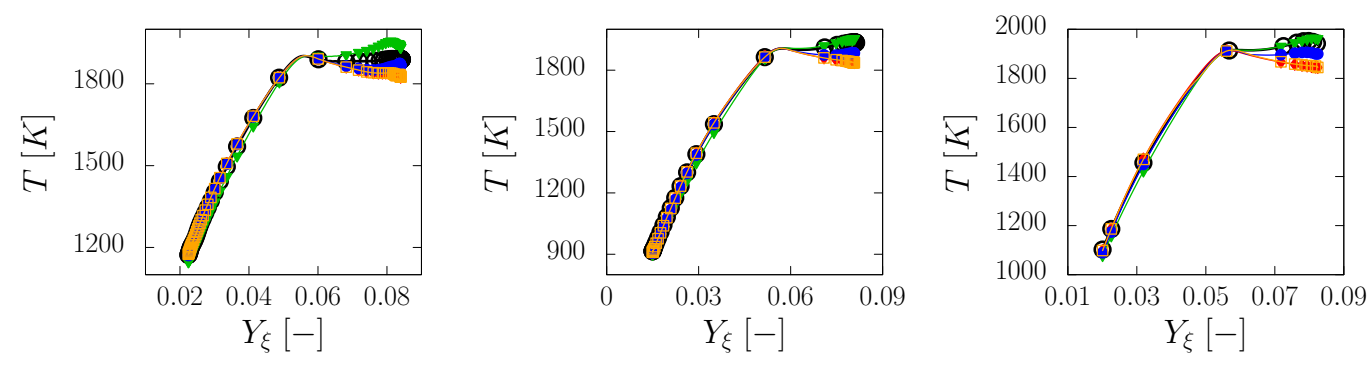

(c) $c=0.9$

Figure 11.: Temperature profiles plotted versus the mixture fraction variable as obtained along normalized progress variable iso-lines for the three cases: Case A: low (left), Case B: moderate (middle), and Case $\mathrm{C}$ : high (right) inlet mixture fraction gradient cases. 

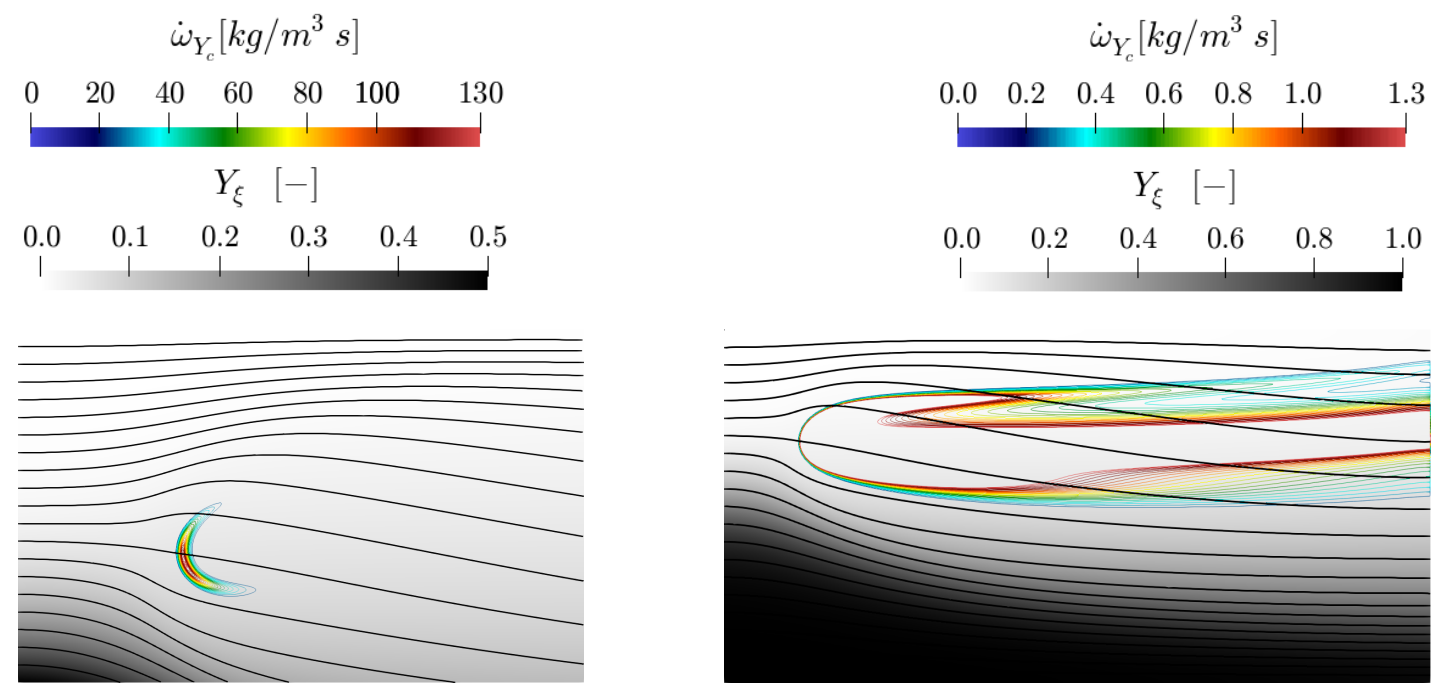

(a) DC
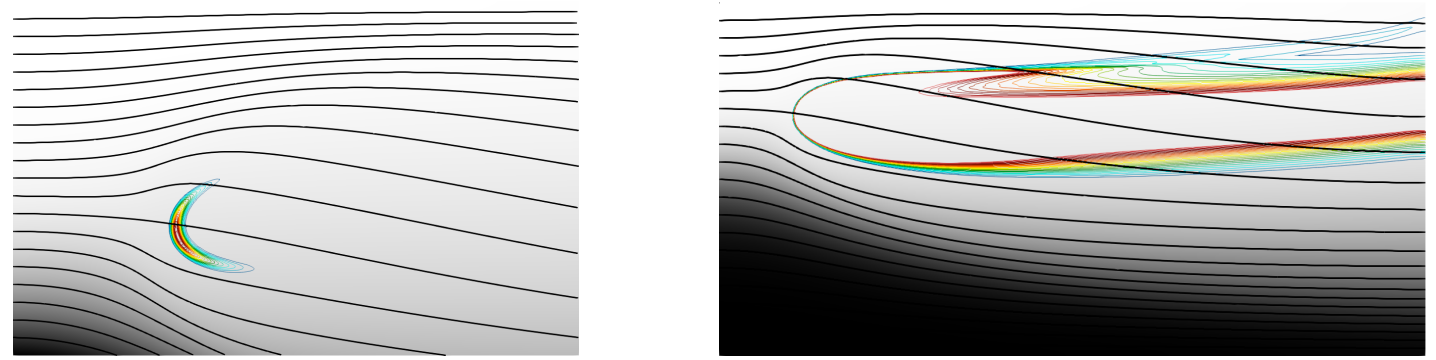

(b) TPF
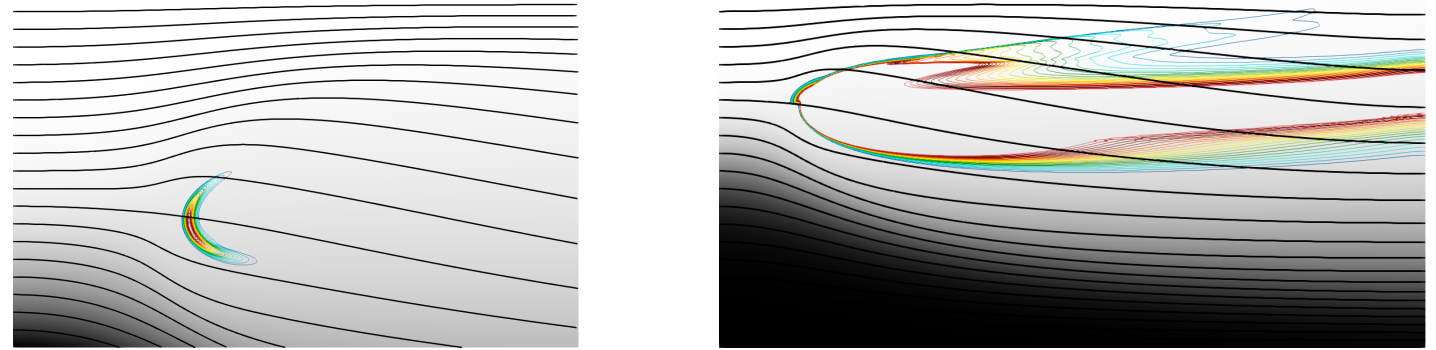

(c) PTF
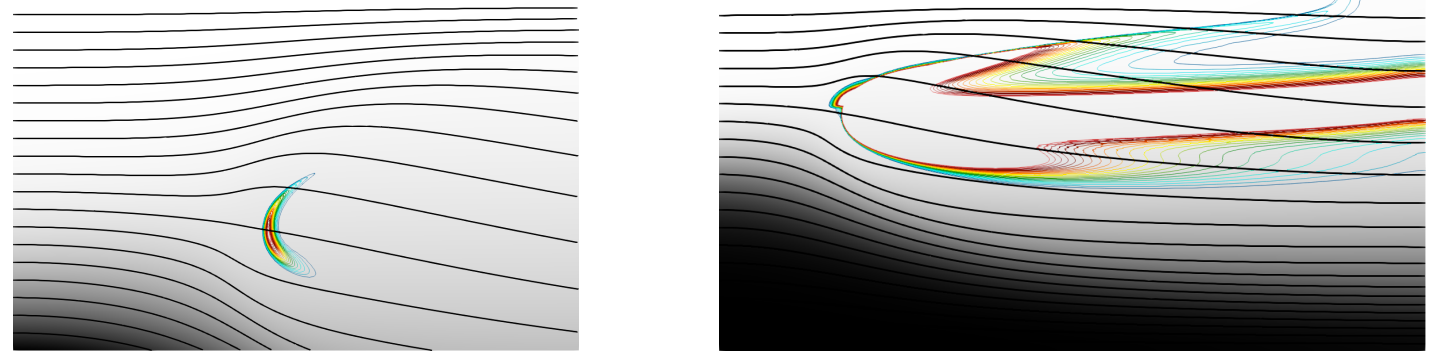

(d) TDF

Figure 12.: Progress variable source term distributions $\dot{\omega}_{Y_{c}}$ associated to the partiallypremixed flame front leading edge (left) and post-diffusion flame (right) superimposed on flow streamlines. 
these results that the prediction of $\mathrm{CO}$ profiles remains a challenging target. To conclude with the triple flame analysis, the profiles of $\mathrm{OH}$ and $\mathrm{CH}_{3}$ mass fractions are plotted in Fig. 14. From a general viewpoint, the TPF model leads to a better level of agreement with the reference DC solution than the TDF model, for the two species under consideration. For the hydroxyl radical mass fraction $Y_{\mathrm{OH}}$, the proposed flame partitioning model (i.e., PTF model) leads to results similar to those obtained with the TPF model, while the computational results are even improved for the methyl radical mass fraction $Y_{\mathrm{CH}_{3}}$.

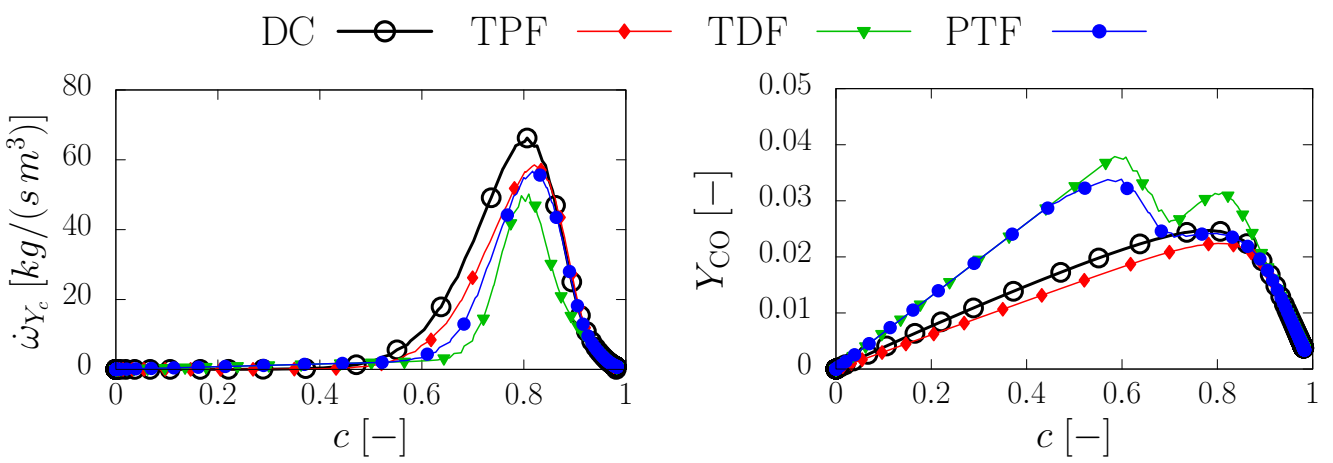

(a) $Y_{\xi}=0.04$
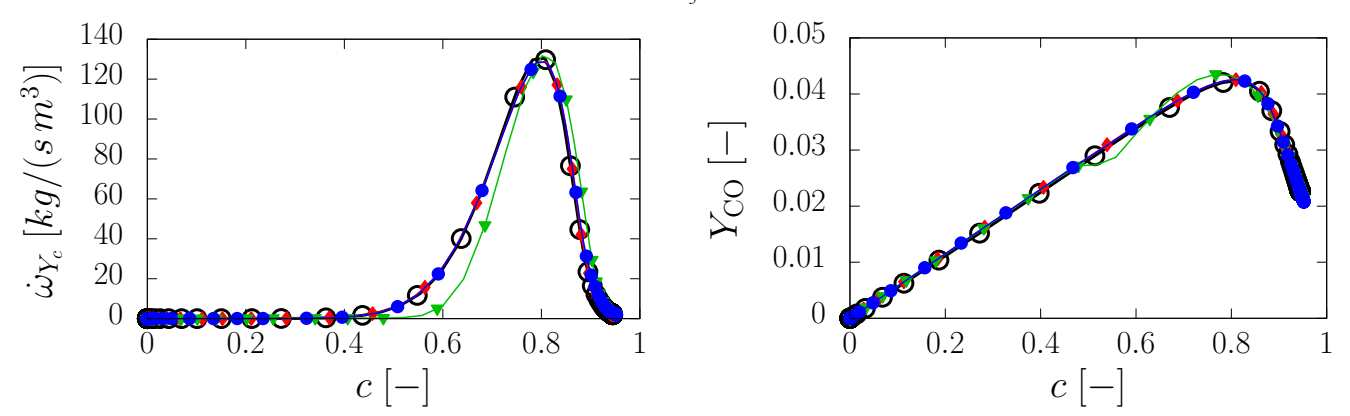

(b) $Y_{\xi}=0.055$
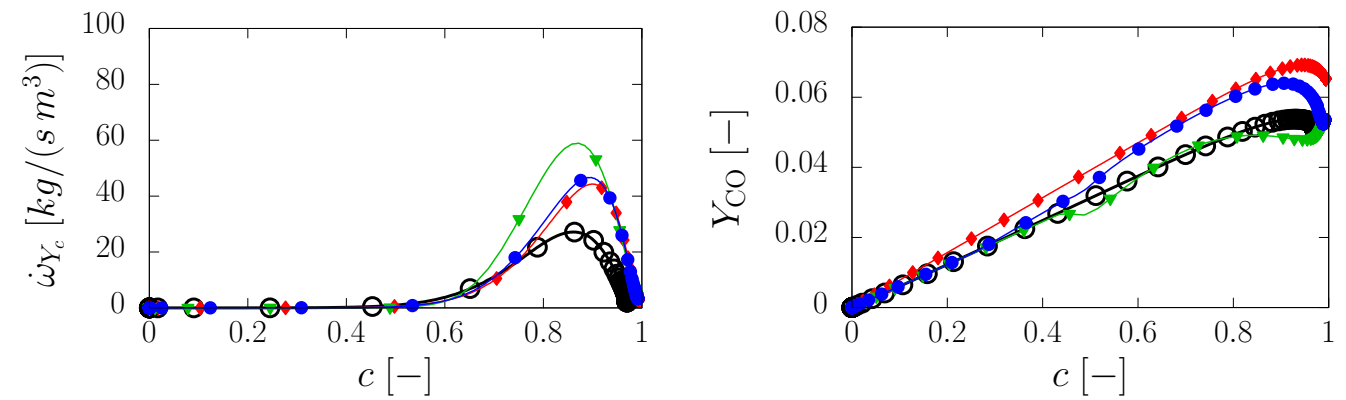

(c) $Y_{\xi}=0.07$

Figure 13.: Profiles of progress variable source term $\dot{\omega}_{Y_{c}}$ and $\mathrm{CO}$ mass fraction $Y_{\mathrm{CO}}$ obtained along mixture fraction iso-lines and plotted versus the normalized progress variable.

From a general point of view, the present set of results, including those issued from the $a$ priori analysis, confirms that the proposed PTF model leads to a satisfactory level of agreement with the reference DC results. 


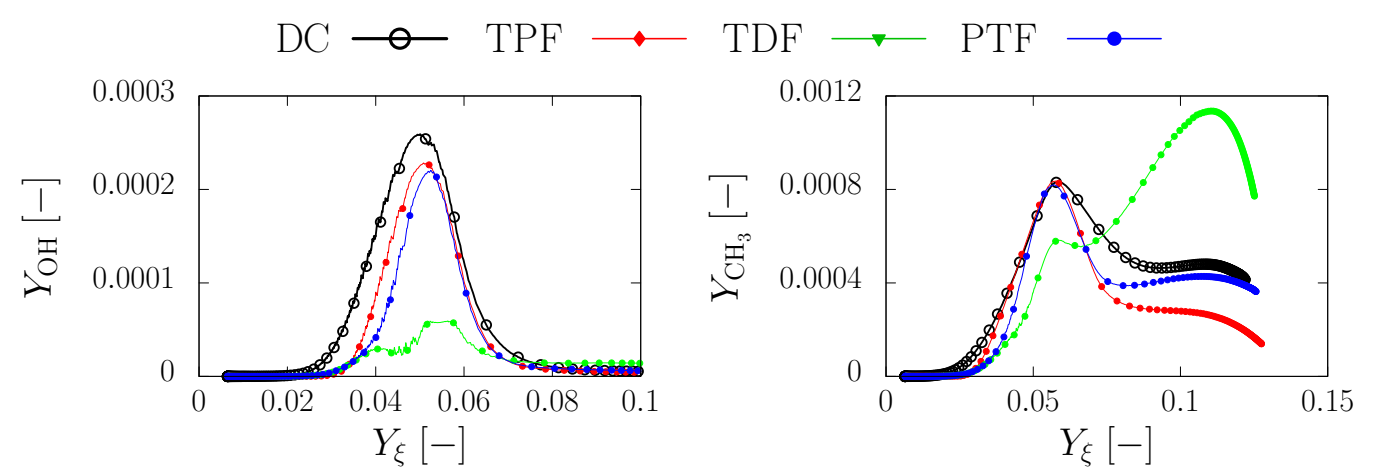

(a) $c=0.6$
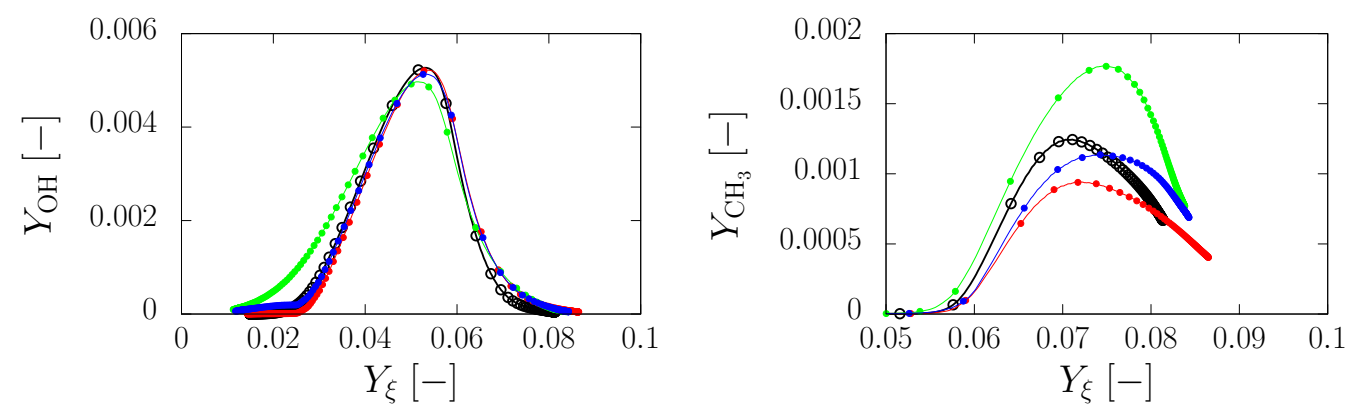

(b) $c=0.9$

Figure 14.: Hydroxyl and methyl mass fractions profiles along normalized progress variable iso-lines for the moderate mixture fraction gradient case.

\subsection{Unsteady flame kernel growth}

The last test case deals with the development of a flame kernel in a non-homogeneous mixture of fresh reactants [4]. In contrast to the two previous cases, the objective is now to evaluate the relevance of the proposed flame index partitioning in a transient situation.

The studied geometry corresponds to a two-dimensional squared computational domain featuring dimensions $L_{x}=L_{y}=L=10 \mathrm{~mm}$. Outlet conditions are imposed on all boundaries and the pressure level is hold constant in the whole computational domain. It is discretized with a uniform Cartesian grid featuring $400 \times 400$ computational cells, which corresponds to a resolution $\Delta x=\Delta y=\Delta=\delta_{L}^{0} / 20$.

Three distinct cases with a different initial equivalence ratio probability density function (PDF) are considered. The corresponding equivalence ratio distributions have been obtained from a single normalized scalar field bounded between zero and unity. Thus, they feature the same spatial distribution, i.e., only their ranges of variations $\left[\Phi_{\min }, \Phi_{\max }\right]$ differ from one case to another. The equivalence ratio distribution retained for the first case (case I) is displayed in Fig. 15. It corresponds to $\Phi$ in the range $[0.5,1.5]$. The other two cases (cases II and III) are generated by rescaling the normalized scalar field in such a manner that the equivalence ratio distribution lies within the following ranges $\Phi \in[0.0,2.0]$ in case II and $\Phi \in[0.0,6.0]$ in case III. It is noteworthy that the mean value of the equivalence ratio associated to the three cases are $\bar{\Phi}=1.0$ (case I), $\bar{\Phi}=1.0$ (case II), and $\bar{\Phi}=3.0$ (case III). The initial velocity field is set to zero (i.e., no velocity). As emphasized in reference [4], the purpose of such a test case is to evaluate the ability to recover a satisfactory development of the flame kernel in a stratified medium and not to describe the ignition and early birth of the initial kernel. Therefore, a flame kernel is initialized in the center of the computational domain. The 
corresponding initial kernel is deposited within an imposed stoichiometric circular region so as to favor its initial growth and avoid extinction as much as possible.

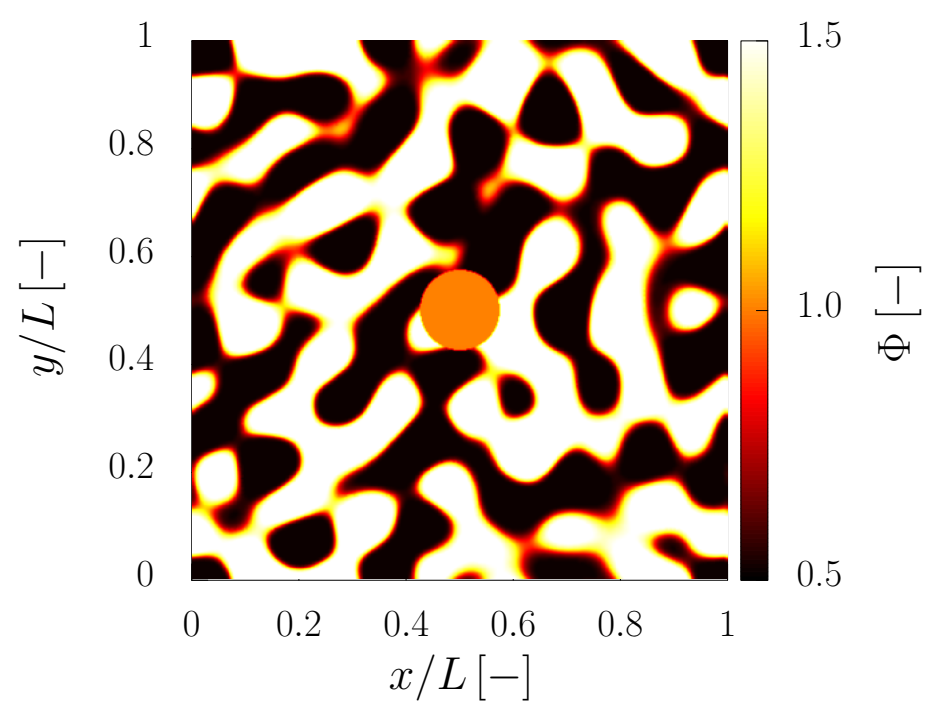

Figure 15.: Initial equivalence ratio distribution together with the stoichiometric patch used to favor the initial growth of the flame kernel.

The flame front development is followed thanks to a progress variable iso-contour of $c=0.3$. It is also used to perform the computation of flame surface averaged quantities, i.e., quantities that are averaged along the corresponding iso-line. For instance, the temporal evolution of the flame front averaged mixture fraction value as well as its gradient, as obtained from the DC computations, are plotted in Fig. 16. The flame front develops in the central stoichiometric region until a peak is reached for the corresponding norm of the mixture fraction gradient. Then, the flame front averaged value of the mixture fraction evolves and progressively approaches the overall mean value.
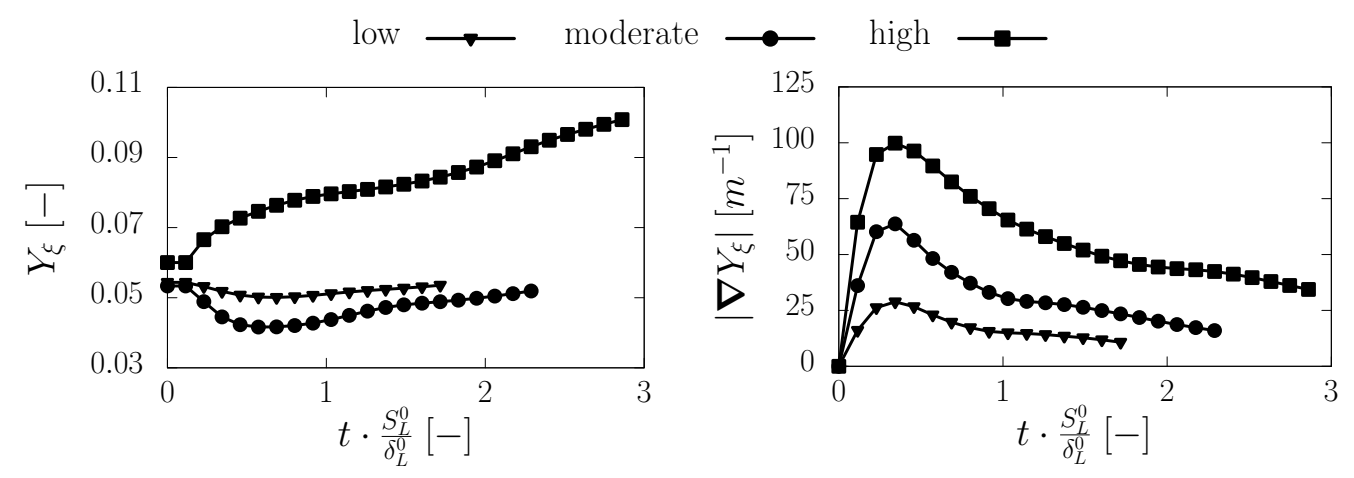

Figure 16.: Temporal evolution of the flame-surface averaged mixture fraction value (left) and associated magnitude of its gradient (right) for the three different cases as obtained from the DC computations

Comparisons of flame front contours and temperature distribution - plotted together with the mixture fraction and progress variable iso-lines - issued from DC and tabulated flamelets 

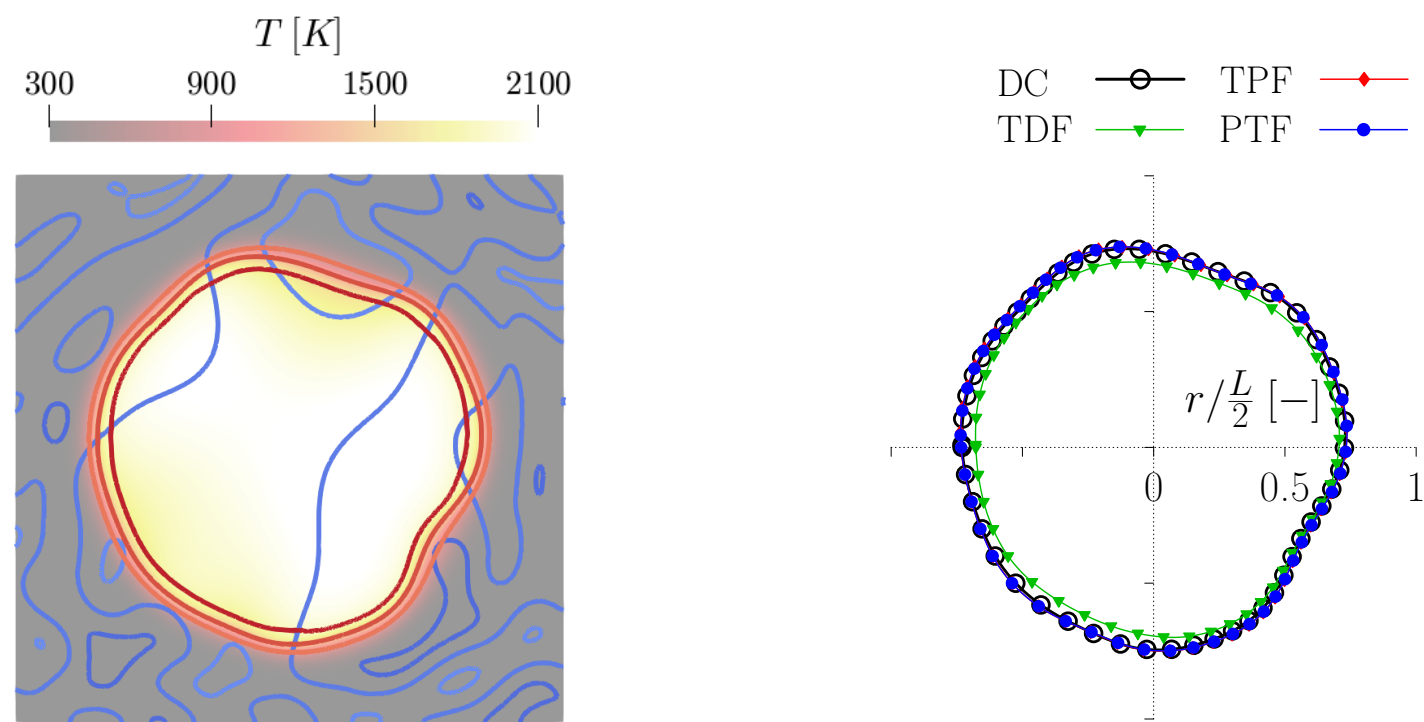

(a) $\Phi \in[0.5,1.5]$
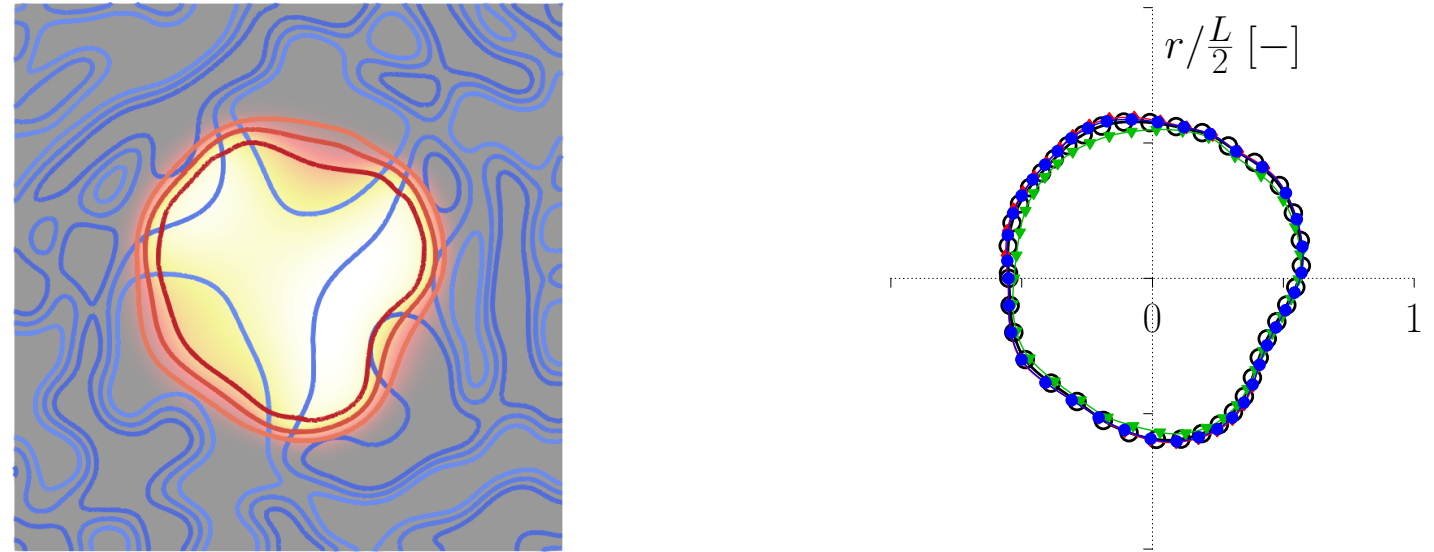

(b) $\Phi \in[0.0,2.0]$
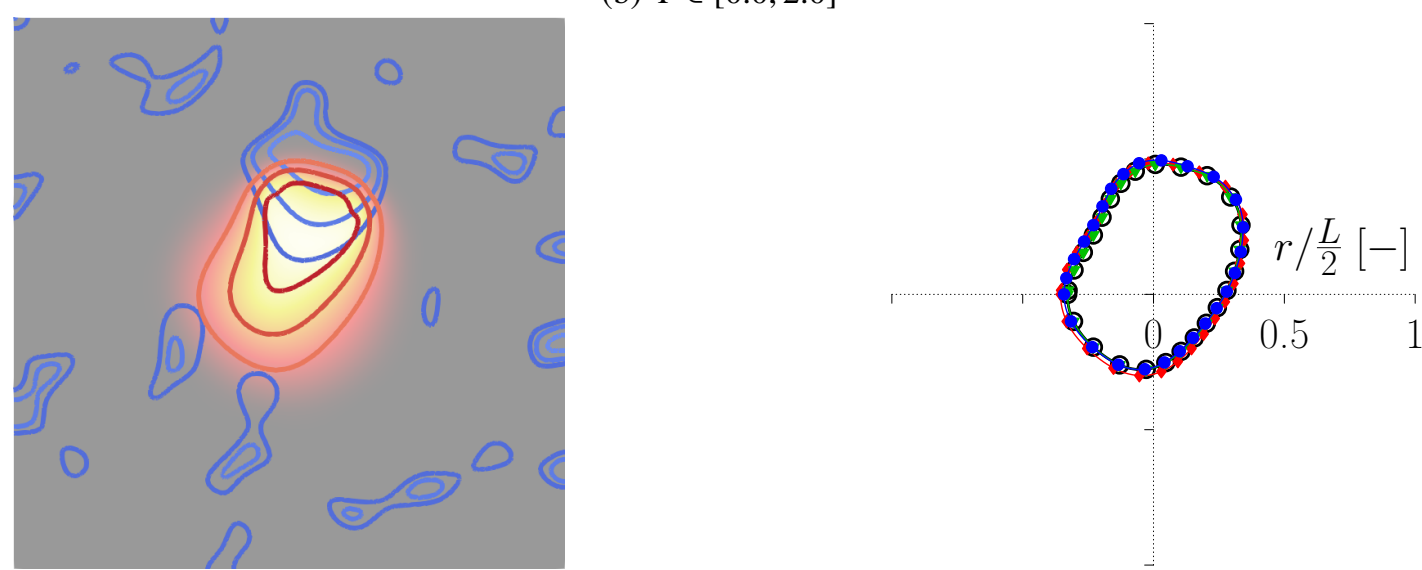

(c) $\Phi \in[0.0,6.0]$

Figure 17.: Temperature field obtained at $t^{*}=1.5$ with the DC representation together with mixture fraction $Y_{\xi}^{*}=\{0.04,0.055,0.07\}$ and progress variable $c^{*}=\{0.3,0.6,0.9\}$ iso-lines (left) ; corresponding flame front contours, i.e., progress variable iso-line $c=0.3$ (right). 

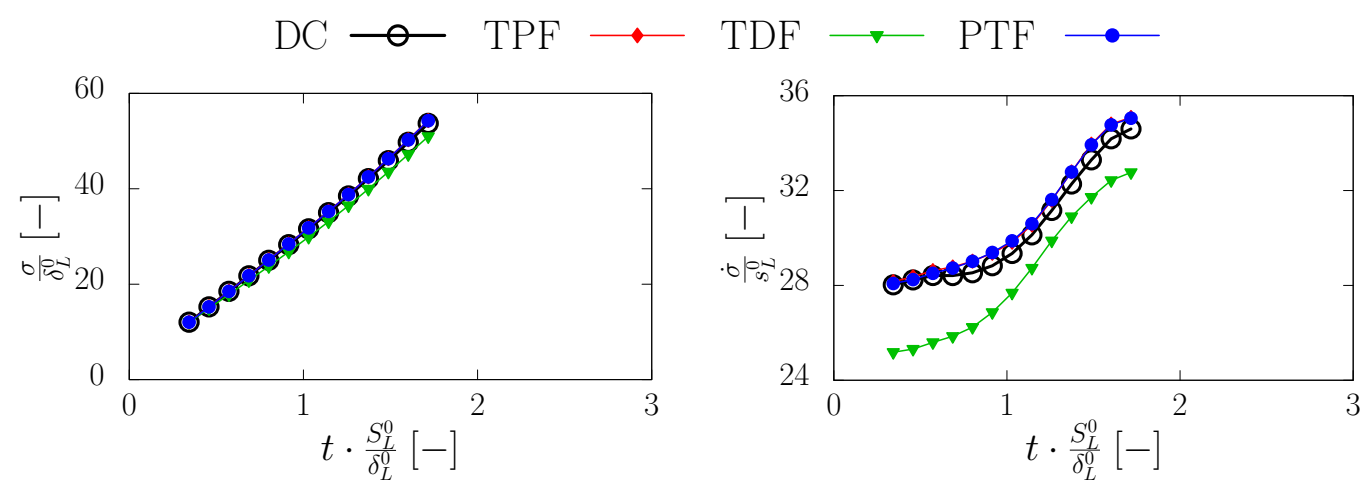

(a) $\Phi \in[0.5,1.5]$ (case I)
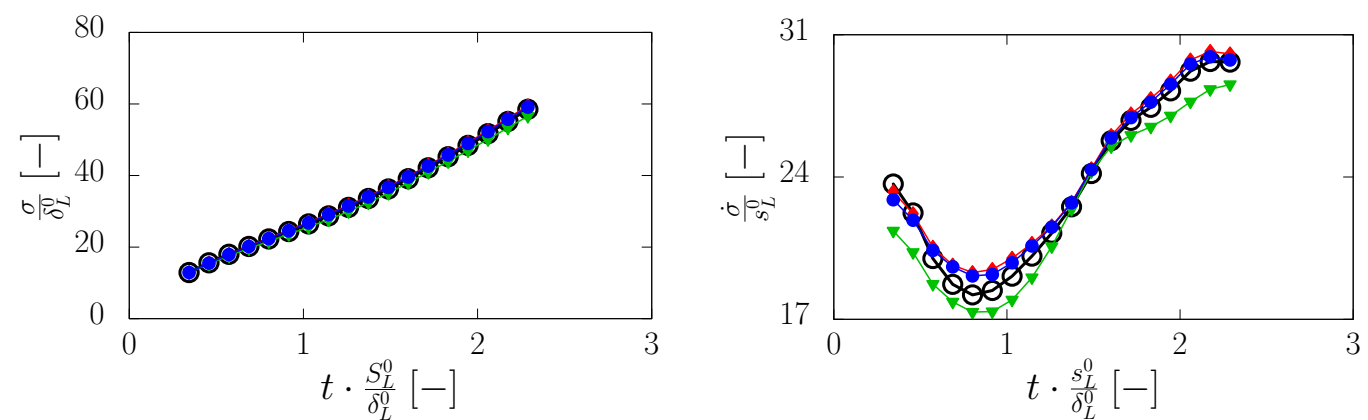

(b) $\Phi \in[0.0,2.0]$ (case II)
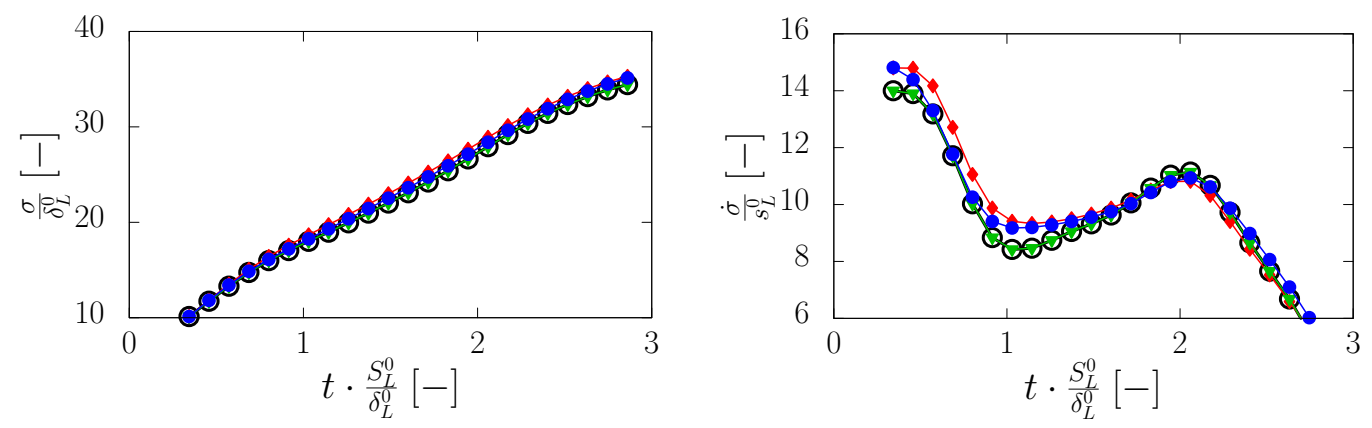

(c) $\Phi \in[0.0,6.0]$ (case III)

Figure 18.: Temporal evolution of the flame front area per unit width (left) and its gradient (right) 
models computations are reported at $t^{*}=1.5$ in Fig. 17. At this time, the field of the mixture fraction obtained for case I is almost fully premixed and the flame front shape differs only slightly from a circular shape. This is in contrast with case II, which displays a more inhomogeneous distribution of the equivalence ratio, although a premixed combustion regime still dominates. There are some non-negligible local variations of the flame propagation speed induced by the inhomogeneous equivalence ratio distribution along the flame surface, which induces some flame wrinkling effects. Finally, case III is diffusion-dominated and there is no perceptible flame wrinkling effects: the three models lead to a similar level of agreement with the reference DC results. These observations are assessed through the comparison of the normalized progress variable iso-line reported on the right side of Fig. 17. While the premixed solution matches well the detailed chemistry (DC) solution over the whole flame front in case I, larger discrepancies are found in case II, even though the flame kernel development is slower. These plots also confirms that the diffusion flame solution provides a good approximation of the DC results in case III.

As emphasized by Er-raiy et al. [4], a typical estimate of the flame front propagation velocity can be deduced from the evolution of the flame surface area or its length $\sigma$ in twodimensional situations, such as the one considered here, and the associated growth rate $\dot{\sigma}$, which are displayed in Fig. 18. In this figure, all quantities are normalized by the premixed laminar flame characteristics at stoichiometry. Case I is associated to a narrow range of variations of the equivalence ratio $\Phi$. In this case, the growth rate $\dot{\sigma}$ increases monotonically with time and there is a significant change of the corresponding positive slope for $t^{*}$ approximately equal to unity. For cases II and III, the flame front first decelerates until $t^{*}$ reaches approximately 0.8 and $\dot{\sigma}$ subsequently increases. This result is an outcome of the increase of the equivalence ratio in regions that were initially very lean. In case III the growth rate $\dot{\sigma}$ decays significantly for $t^{*}>2.0$ since molecular mixing processes take place to bring the whole field of the mixture fraction towards its mean value (i.e., $\bar{\Phi}=3.0$ ), which lies beyond the rich flammability limit (i.e., $\Phi=2.75$ for the present mixture). Additional results, which are not reported here just for the sake of conciseness, do show that further increases of the global equivalence ratio through an extension of $\Phi_{\max }$ further decreases the initial flame kernel propagation velocity.

Special attention is now paid to the behaviour of the different models. In case I the corresponding propagation speed appears to be clearly under-estimated with the TDF model, whereas the TPF model is able to capture the propagation velocity fairly well. The flame index modelling also correlates well with the reference DC results: the flame index value at the flame front location is close to unity. For case II, the results obtained with the proposed flame index partition are slightly better than those obtained with tabulated premixed flamelets, especially around $t^{*}=0.9$ and $t^{*}=2$. For an increased range of variations of the equivalence ratio $\Phi$, and despite the difference between the results obtained with tabulated premixed and tabulated diffusion flamelets remain rather moderate when compared to the previous cases, the diffusion model leads to better levels of agreement with the reference DC solution.

Finally the whole set of computational results confirms that the present multiregime flamelet model is able to identify the relevant regime in the case of combustion of inhomogeneous fuel-air mixtures.

\section{Conclusions and future works}

A new definition of the premixedness index is introduced for partially premixed combustion. It is based on a weighted form of the cross-scalar dissipation rate of the mixture fraction $Y_{\xi}$ and progress variable $Y_{c}$. The corresponding multi-regime flamelet framework is used in con- 
junction with tabulations of the two elementary and archetypal flame prototypes associated to one-dimensional laminar premixed flame and counterflow laminar diffusion flame. The corresponding prototypes indeed remain the cornerstone and reference building blocks of most combustion models. The resulting modelling framework is used to proceed with the numerical simulation of three successive validation subsets: (i) counterflow flames, (ii) stabilized triple flames, and (iii) unsteady flame kernel developments in non-homogeneous mixtures of fresh reactants. The validation procedure is based on both a priori and a posteriori analyses. In both analyses, the comparisons performed between the results obtained with the proposed flame partitioning and reference detailed chemistry computations lead to a good level of agreement. To conclude, it seems worth emphasizing that this flame index does not require the consideration of any other quantities in addition to those already used to parameterize the flamelets databases, i.e., $Y_{\xi}$ and $Y_{c}$, the transport equations of which are solved during the numerical simulations. Existing databases of tabulated premixed and diffusion flamelets can be directly sed, and weighted with the premixedness index definition that is introduced in the present study. The model has been applied with a simple definition of the progress variable and leads to an improved level of agreement with the reference DC computations in comparison to the results issued from the consideration of a single flamelet databases (TPF or TDF). Since some modelling efforts have already been spent to represent the first- and second-order moments of the corresponding fluctuations, see for instance references [28[45], the present approach can be readily extended to the consideration of turbulent reactive flows of partially premixed reactants. Thus, the model based on the new flame index proposal appears as a good candidate for future applications to turbulent combustion modelling.

\section{Acknowledgements}

745 Funding: The research leading to these results has received funding through the Spanish Ministry of Economy and Competitiveness in the frame of the CHEST project (TRA201789139-C2-2-R).

Conflict of Interest: Daniel Mira acknowledges the Juan de la Cierva personal grant IJCI2015-26686.

\section{Appendix A. Validation of the Cantera implementation}

To make sure that the finite rate solver provides an appropriate reference solution for the comparison with the different flamelet models, one-dimensional results issued from its implementation in OpenFoam are compared to the Cantera results from which the tables are generated. Two freely-propagating laminar premixed flames and two laminar counterflow diffusion flames are considered.

Figure A1 confirms that the temperature and $\mathrm{CO}$ mass fraction obtained for the premixed flamelets are in excellent agreement with the reference Cantera solutions for the equivalence ratios considered. Conversely, the DC model leads to a slightly higher value of both $T$ and $Y_{\mathrm{CO}}$. This may be an outcome of the gradients of velocity, chemical species mass fractions, and enthalpy which are set to zero at the inlet boundaries in OpenFoam computations, while they are not in Cantera computations. This leads to higher SDR values in OpenFOAM, thus inducing the small observed discrepancies. 

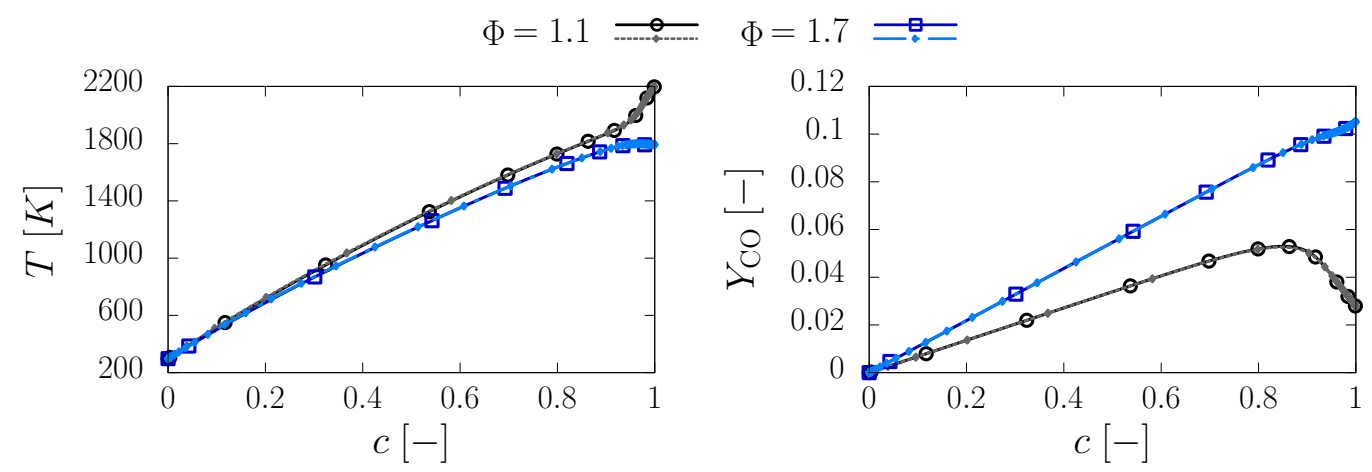

(a) Premixed flamelets
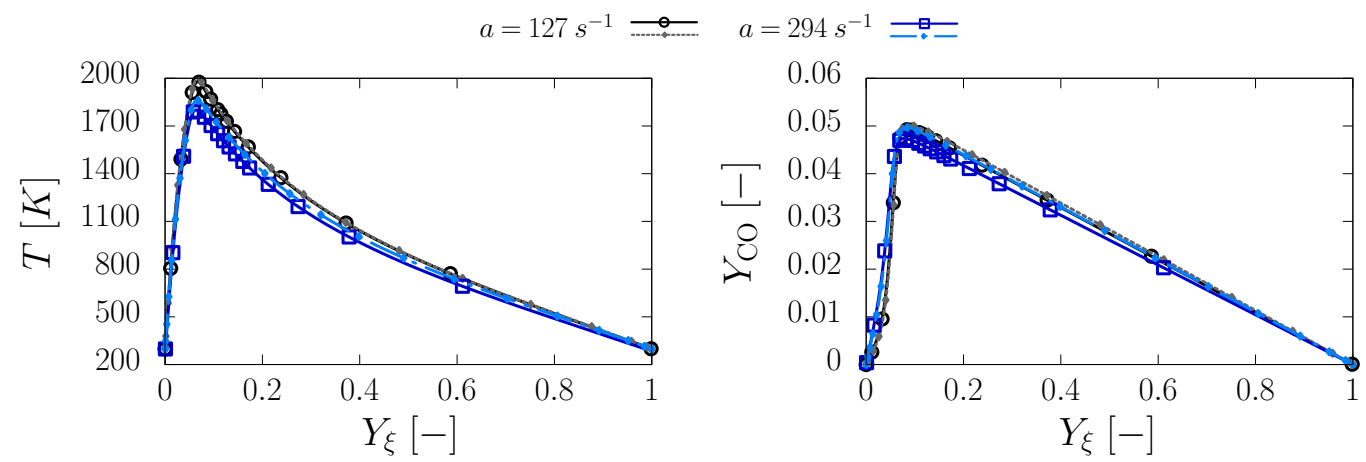

(b) Diffusion flamelets

Figure A1.: Profiles of temperature and CO mass fraction obtained for one-dimensional premixed and counter-flow diffusion flamelets using the finite rate solver implementation in OpenFoam (solid line) and Cantera (dashed line)

\section{References}

[1] E. Fernández-Tarrazo, A.L. Sánchez, A. Linán, and F.A. Williams, A simple one-step chemistry model for partially premixed hydrocarbon combustion, Combustion and Flame 147 (2006), pp. 32-38.

[2] B. Franzelli, E. Riber, M. Sanjosé, and T. Poinsot, A two-step chemical scheme for kerosene-air premixed flames, Combustion and Flame 157 (2010), pp. 1364-1373.

[3] P. Boivin, C. Jiménez, A.L. Sánchez, and F.A. Williams, A four-step reduced mechanism for syngas combustion, Combustion and Flame 158 (2011), pp. 1059-1063.

[4] A. Er-raiy, Z. Bouali, J. Reveillon, and A. Mura, Optimized single-step (OSS) chemistry models for the simulation of turbulent premixed flame propagation, Combustion and Flame 192 (2018), pp. $130-148$.

[5] J.A. van Oijen and L.P.H. de Goey, Modelling of premixed laminar flames using flameletgenerated manifolds, Combustion Science and Technology 161 (2001), pp. 113-137.

[6] O. Gicquel, N. Darabiha, and D. Thévenin, Laminar premixed hydrogen/air counterflow flame simulations using flame prolongation of ILDM with differential diffusion, Proceedings of the Combustion Institute 28 (2000), pp. 1901-1908.

[7] J.A. van Oijen and L.P.H. de Goey, A numerical study of confined triple flames using a flameletgenerated manifold, Combustion Theory and Modelling 8 (2004), pp. 141-163.

[8] B. Fiorina, O. Gicquel, L. Vervisch, S. Carpentier, and N. Darabiha, Approximating the chemical structure of partially premixed and diffusion counterflow flames using FPI flamelet tabulation, Combustion and Flame 140 (2005), pp. 147-160.

[9] S. Gövert, D. Mira, J.B.W. Kok, M. Vázquez, and G. Houzeaux, Turbulent combustion modelling 
of a confined premixed jet flame including heat loss effects using tabulated chemistry, Applied Energy 156 (2015), pp. $804-815$.

[10] D. Mira, O. Lehmkuhl, A. Both, P. Stathopoulos, T. Tanneberger, T. Reichel, C. Paschereit, M. Vazquez, and G. Houzeaux, Numerical characterization of a premixed hydrogen flame under conditions close to flashback, Flow Turbulence and Combustion In press (2020).

[11] N. Peters, Laminar diffusion flamelet models in non-premixed turbulent combustion, Progress in Energy and Combustion Science 10 (1984), pp. 319-339.

[12] C.D. Pierce, Progress-variable approach for large-eddy simulation of turbulent combustion, Ph.D. diss., Stanford University, 2001.

[13] C.D. Pierce and P. Moin, Progress-variable approach for large-eddy simulation of non-premixed turbulent combustion, Journal of Fluid Mechanics 504 (2004), pp. 73—97.

[14] B. Franzelli, B. Fiorina, and N. Darabiha, A tabulated chemistry method for spray combustion, Proceedings of the Combustion Institute 34 (2013), pp. 1659-1666.

[15] X. Wen, X.S. Bai, K. Luo, H. Wang, Y. Luo, and J. Fan, A generalized flamelet tabulation method for partially premixed combustion, Combustion and Flame 198 (2018), pp. 54-68.

[16] E. Knudsen and H. Pitsch, A general flamelet transformation useful for distinguishing between premixed and non-premixed modes of combustion, Combustion and Flame 156 (2009), pp. 678696.

[17] J. van Oijen, A. Donini, R. Bastiaans, J. ten Thije Boonkkamp, and L. de Goey, State-of-the-art in premixed combustion modeling using flamelet generated manifolds, Progress in Energy and Combustion Science 57 (2016), pp. 30-74.

[18] T. Lu, C. Yoo, J. Chen, and C. Law, Three-dimensional direct numerical simulation of a turbulent lifted hydrogen jet flame in heated coflow: a chemical explosive mode analysis, Journal of Fluid Mechanics 652 (2010), pp. 45-64.

[19] S. Hartl, D. Geyer, A. Dreizler, G. Magnotti, R. Barlow, and C. Hasse, Regime identification from raman/rayleigh line measurements in partially premixed flames, Combustion and Flame 189 (2018), pp. 126-141.

[20] H. Yamashita, M. Shimada, and T. Takeno, A numerical study on flame stability at the transition point of jet diffusion flames, Symposium (International) on Combustion 26 (1996), pp. 27-34.

[21] Y. Mizobuchi, S. Tachibana, J. Shinio, S. Ogawa, and T. Takeno, A numerical analysis of the structure of a turbulent hydrogen jet lifted flame, Proceedings of the Combustion Institute 29 (2002), pp. 2009-2015.

[22] Y. Mizobuchi, J. Shinjo, S. Ogawa, and T. Takeno, A numerical study on the formation of diffusion flame islands in a turbulent hydrogen jet lifted flame, Proceedings of the Combustion Institute 30 (2005), pp. 611-619.

[23] R. Buttay, L. Gomet, G. Lehnasch, and A. Mura, Highly resolved numerical simulation of combustion downstream of a rocket engine igniter, Shock Waves 27 (2017), pp. 655-674.

[24] S. Zhao, Z. Bouali, and A. Mura, Computational investigation of weakly turbulent flame kernel growths in iso-octane droplet clouds in CVC conditions, Flow, Turbulence and Combustion 104 (2020), pp. 139-177.

[25] D. Lignell, J. Chen, and H. Schmutz, Effects of Damköhler number on flame extinction and reignition in turbulent non-premixed flames using DNS, Combustion and Flame 158 (2011), pp. 949963.

[26] A. Abdelsamie and D. Thévenin, Direct numerical simulation of spray evaporation and autoignition in a temporally-evolving jet, Proceedings of the Combustion Institute 36 (2017), pp. 24932502.

[27] K.N.C. Bray, P. Domingo, and L. Vervisch, Role of the progress variable in models for partially premixed turbulent combustion, Combustion and Flame 141 (2005), pp. 431-437.

[28] A. Mura, V. Robin, and M. Champion, Modeling of scalar dissipation in partially premixed turbulent flames, Combustion and Flame (2007), pp. 217-224.

[29] J. Lamouroux, M. Ihme, B. Fiorina, and O. Gicquel, Tabulated chemistry approach for diluted combustion regimes with internal recirculation and heat losses, Combustion and Flame 161 (2014), pp. 2120-2136.

[30] A. Pires da Cruz, A.M. Dean, and J.M. Grenda, A numerical study of the laminar flame speed of 
stratified methane/air flames, Proceedings of the Combustion Institute 28 (2000), pp. 1925-1932.

[31] D. Barré, L. Esclapez, M. Cordier, E. Riber, B. Cuenot, G. Staffelbach, B. Renou, A. Vandel, L.Y. Gicquel, and G. Cabot, Flame propagation in aeronautical swirled multi-burners: Experimental and numerical investigation, Combustion and Flame 161 (2014), pp. 2387-2405.

[32] D.A. Rosenberg, P.M. Allison, and J.F. Driscoll, Flame index and its statistical properties measured to understand partially premixed turbulent combustion, Combustion and Flame 162 (2015), pp. $2808-2822$.

[33] P.J.M. Ferrer, G. Lehnasch, and A. Mura, Compressibility and heat release effects in high-speed reactive mixing layers $i$. structure of the stabilization zone and modeling issues relevant to turbulent combustion in supersonic flows, Combustion and Flame 180 (2017), pp. 304-320.

[34] D. Butz, S. Hartl, S. Popp, S. Walther, R.S. Barlow, C. Hasse, A. Dreizler, and D. Geyer, Local flame structure analysis in turbulent ch4/air flames with multi-regime characteristics, Combustion and Flame 210 (2019), pp. 426-438.

[35] D.G. Goodwin, H.K. Moffat, and R.L. Speth, Cantera: an object-oriented software toolkit for chemical kinetics, thermodynamics, and transport processes, http://www.cantera.org (2017). Version 2.3.0.

[36] University of California at San Diego, Chemical-Kinetic Mechanisms for Combustion Applications, San Diego Mechanism web page http://combustion.ucsd.edu Publication date: 15 August 2016.

[37] R.W. Bilger, S.H. Starner, and R.J. Kee, On reduced mechanisms for methane-air combustion in nonpremixed flames, Combustion and Flame 80 (1990), pp. 135-149.

[38] L. Gomet, V. Robin, and A. Mura, A multiple-inlet mixture fraction model for nonpremixed combustion, Combustion and Flame 162 (2015), pp. 668-687.

[39] A.W. Vreman, B.A. Albrecht, J.A. van Oijen, L.P.H. de Goey, and R.J.M. Bastiaans, Premixed and nonpremixed generated manifolds in large-eddy simulation of sandia flame $D$ and $F$, Combustion and Flame 153 (2008), pp. 394-416.

[40] T. Fiala and T. Sattelmayer, Nonpremixed counterflow flames: Scaling rules for batch simulations, Journal of Combustion 7 (2014).

[41] E. Knudsen and H. Pitsch, Capabilities and limitations of multi-regime flamelet combustion models, Combustion and Flame 159 (2012), pp. 242-264.

[42] G.R. Ruetsch, L. Vervisch, and A. Liñán, Effects of heat release on triple flames, Physics of Fluids 7 (1995).

[43] P. Domingo and L. Vervisch, Triple flames and partially premixed combustion in autoignition of non-premixed turbulent mixtures, Symposium (International) on Combustion 26 (1996), pp. $233-240$.

[44] H. Wu, Y.C. See, Q. Wang, and M. Ihme, A pareto-efficient combustion framework with submodel assignment for predicting complex flame configurations, Combustion and Flame 162 (2015), pp. 4208-4230.

[45] V. Robin, A. Mura, M. Champion, O. Degardin, B. Renou, and M. Boukhalfa, Experimental and numerical analysis of stratified turbulent V-shaped flames, Combustion and Flame 153 (2008), pp. 288-315. 